\title{
PLANE WAVE STABILITY OF THE SPLIT-STEP FOURIER METHOD FOR THE NONLINEAR SCHRÖDINGER EQUATION
}

\author{
ERWAN FAOU ${ }^{1,2}$, LUDWIG GAUCKLER ${ }^{3}$ and CHRISTIAN LUBICH ${ }^{4}$ \\ ${ }^{1}$ INRIA and ENS Cachan Bretagne, Avenue Robert Schumann, F-35170 Bruz, France; \\ email: Erwan.Faou@inria.fr \\ ${ }^{2}$ Département de mathématiques et applications, École normale supérieure, \\ 45 rue d'Ulm, F-75230 Paris Cedex 05, France \\ ${ }^{3}$ Institut für Mathematik, Technische Universität Berlin, Straße des 17. Juni 136, \\ D-10623 Berlin, Germany; \\ email: gauckler@math.tu-berlin.de \\ ${ }^{4}$ Mathematisches Institut, Universität Tübingen, Auf der Morgenstelle 10, \\ D-72076 Tübingen, Germany; \\ email: lubich@na.uni-tuebingen.de
}

Received 4 June 2013; accepted 13 February 2014

\begin{abstract}
Plane wave solutions to the cubic nonlinear Schrödinger equation on a torus have recently been shown to behave orbitally stable. Under generic perturbations of the initial data that are small in a high-order Sobolev norm, plane waves are stable over long times that extend to arbitrary negative powers of the smallness parameter. The present paper studies the question as to whether numerical discretizations by the split-step Fourier method inherit such a generic long-time stability property. This can indeed be shown under a condition of linear stability and a nonresonance condition. They can both be verified in the case of a spatially constant plane wave if the time step-size is restricted by a Courant-Friedrichs-Lewy condition (CFL condition). The proof first uses a Hamiltonian reduction and transformation and then modulated Fourier expansions in time. It provides detailed insight into the structure of the numerical solution.
\end{abstract}

2010 Mathematics Subject Classification: primary 65P10, 65P40; secondary 65M70

(c) The Author(s) 2014. The online version of this article is published within an Open Access environment subject to the conditions of the Creative Commons Attribution licence $<$ http://creativecommons.org/licenses/by/3.0/>. 


\section{Introduction}

We consider the cubic nonlinear Schrödinger equation

$$
i \frac{\partial}{\partial t} u=-\Delta u+\lambda|u|^{2} u, \quad u=u(x, t),
$$

in the defocusing $(\lambda=+1)$ or focusing $(\lambda=-1)$ case. We impose periodic boundary conditions in arbitrary spatial dimension $d \geqslant 1$ : the spatial variable $x$ belongs to the $d$-dimensional torus $\mathbb{T}^{d}=\mathbb{R}^{d} /(2 \pi \mathbb{Z})^{d}$.

This nonlinear Schrödinger equation has a class of simple solutions, the plane wave solutions

$$
u(x, t)=\rho e^{i(\ell \cdot x-\omega t)}
$$

for $\rho \geqslant 0, \ell \in \mathbb{Z}^{d}$, and $\omega=|\ell|^{2}+\lambda \rho^{2}$, where $\ell \cdot x=\ell_{1} x_{1}+\cdots+\ell_{d} x_{d}$ and $|\ell|^{2}=\ell \cdot \ell$. A natural question is whether these plane wave solutions (2) are stable under small perturbations of the initial value. In this context it is common knowledge that a linear stability analysis, where one examines the eigenvalues of the linearization of the nonlinear Schrödinger equation (1) around a plane wave, leads to the condition $1+2 \lambda \rho^{2} \geqslant 0$ for (linear) stability; see for instance [1, Section 5.1.1]. Since nonlinear effects are ignored, the validity of such a linear stability analysis is inherently restricted to a short time interval. Stability and instability on long time intervals of plane waves in the exact solution are discussed in the recent papers $[8,18]$, respectively. Of particular importance for the present paper is [8], where orbital stability over long times is shown for perturbations in high-order Sobolev spaces. Orbital stability means that the solution stays close to the orbit (2).

From the viewpoint of numerical analysis, it is of interest whether (and if so why) a numerical method shares the stability or instability of the exact solution near plane waves. This is the topic of the present paper.

This problem can be traced back to the seminal paper [25] by Weideman and Herbst, from 1986. In that paper, conditions on the discretization parameters for various numerical methods are derived that ensure that the numerical solution shares the linear stability of the exact solution. This is done by examining the eigenvalues of the linearization around a plane wave of a numerical method applied to (1). Such a linear stability analysis has recently been extended to different numerical methods $[4,6,21,22]$.

In the present paper, we take up this line of research. In contrast to previous work [4, 6, 21, 22, 25], however, we are interested in the long-time behaviour of a numerical method near plane waves, and hence a linear stability analysis is of limited use. We pursue the question as to whether the remarkably stable behaviour 
on long time intervals of the exact solution near plane waves [8] is shared by one of the most popular numerical methods for the nonlinear Schrödinger equation, the split-step Fourier method [19]. This method combines a Fourier collocation in space with a Strang splitting in time; see Section 2.1. It integrates plane wave solutions (2) exactly. Our main result states that the long-time orbital stability of the exact solution near plane waves transfers to the numerical solution; see Section 2.2 for a precise statement. In the case of a spatially constant plane wave $(\ell=0$ in (2)), the case considered by Weideman and Herbst [25], it is further shown that the assumptions of this main result essentially hold under a CourantFriedrichs-Lewy condition (CFL condition) on the discretization parameters; see Section 2.3.

The long-time stability result of the present paper deals with the completely resonant equation (1): the eigenvalues (frequencies) of the linear part of the equation are $|j|^{2}, j \in \mathbb{Z}^{d}$, whose integer linear combinations may vanish identically. This is in marked contrast to previous long-time stability results for numerical discretizations of nonlinear Hamiltonian partial differential equations that consider nonresonant situations. See [9-11, 13] for the split-step Fourier method applied to the nonlinear Schrödinger equation, where (1) is considered with an additional (generic but artificial) convolution term $V \star u$ in order to have nonresonant frequencies. Another feature of the result in the present paper is that it covers a much larger class of initial values that are not small than the aforementioned previous stability results that all deal with small initial values.

The proof of our stability result is given in Sections 3-5. We first eliminate, in Section 3, the principal Fourier mode from the numerical scheme with a sequence of transformations and reductions. The resulting system of equations has small initial values. This enables us to use the technique of modulated Fourier expansions for its long-time analysis; see Section 4. It is likely that normal form techniques in the spirit of $[9,10]$ would lead to similar conclusions, but we have not worked out the details. In order to obtain results that are valid on long time intervals, the frequencies have to satisfy a certain nonresonance condition. In fact, the completely resonant frequencies of the nonlinear Schrödinger equation are modified during the transformations of Section 3, and we are able to verify a nonresonance condition for the new frequencies in the final section, Section 5.

\section{Numerical method and statement of the main results}

2.1. The split-step Fourier method. We discretize the nonlinear Schrödinger equation (1) with the split-step Fourier method as introduced in [19, 24, 25]. In this method, the equation is discretized in space by a spectral collocation method and in time by a splitting integrator. 
Discretization in space. For the discretization in space, we make the ansatz

$$
u_{K}(x, t)=\sum_{j \in \mathcal{K}} u_{j}(t) e^{i(j \cdot x)} \quad \text { with } \mathcal{K}:=\{-K, \ldots, K-1\}^{d}
$$

with the spatial discretization parameter $K$. For fixed $t, u_{K}(\cdot, t)$ is a trigonometric polynomial which is uniquely determined by its values in the collocation points $x_{j}=\pi j / K, j \in \mathcal{K}$. Requiring that the ansatz $u_{K}$ fulfills the nonlinear Schrödinger equation (1) in the collocation points leads to the equation

$$
i \frac{\partial}{\partial t} u_{K}=-\Delta u_{K}+\lambda \mathcal{Q}\left(\left|u_{K}\right|^{2} u_{K}\right), \quad u_{K}(\cdot, 0)=\mathcal{Q}(u(\cdot, 0)),
$$

where the trigonometric interpolation $\mathcal{Q}(u)$ (with respect to the spatial variable $x$ ) of a function $u(x)=\sum_{k \in \mathbb{Z}^{d}} u_{k} e^{i(k \cdot x)}$ is the uniquely determined trigonometric polynomial that interpolates $u$ in the collocation points. This trigonometric interpolation is given by

$$
\mathcal{Q}(u)=\sum_{j \in \mathcal{K}} \widetilde{u}_{j} e^{i(j \cdot x)} \quad \text { with } \tilde{u}_{j}=\sum_{k \in \mathbb{Z}^{d}: k \equiv j \bmod 2 K} u_{k},
$$

where the congruence modulo $2 K$ has to be understood entrywise.

Discretization in time. Equation (3) is then discretized in time by a splitting integrator with time step-size $h$. For this purpose, we split (3) into its linear part and its nonlinear part,

$$
i \frac{\partial}{\partial t} u_{K}=-\Delta u_{K} \quad \text { and } \quad i \frac{\partial}{\partial t} u_{K}=\lambda \mathcal{Q}\left(\left|u_{K}\right|^{2} u_{K}\right)
$$

Denoting by $\Phi_{\text {linear }}^{h}$ and $\Phi_{\text {nonlinear }}^{h}$ the flows over a time $h$ of these equations, we compute approximations $u_{K}^{n}$ to $u_{K}\left(\cdot, t_{n}\right)$ at discrete times $t_{n}=n h$ by

$$
u_{K}^{n+1}=\Phi_{\text {linear }}^{h} \circ \Phi_{\text {nonlinear }}^{h}\left(u_{K}^{n}\right) .
$$

The initial value $u_{K}^{0}$ is chosen as

$$
u_{K}^{0}=u_{K}(\cdot, 0)=\mathcal{Q}(u(\cdot, 0)) .
$$

Equations (4) provide a fully discrete scheme for the numerical solution of the nonlinear Schrödinger equation (1), the split-step Fourier method. Since its introduction in [19], it has become a widely used and well-analyzed method; see for example $[1,7,11,20,24,25]$ and the references therein. 
Computational aspects. In (4), both flows $\Phi_{\text {linear }}^{h}$ and $\Phi_{\text {nonlinear }}^{h}$ can be computed exactly in an efficient way. The flow of the linear equation is given in terms of the Fourier coefficients $u_{j}$ of a trigonometric polynomial $u(x)=\sum_{j \in \mathcal{K}} u_{j} e^{i(j \cdot x)}$ by

$$
\Phi_{\text {linear }}^{h}(u)=\sum_{j \in \mathcal{K}} e^{-i|j|^{2} h} u_{j} e^{i(j \cdot x)} .
$$

Thus, it can be computed easily in terms of these Fourier coefficients. On the other hand, the flow of the nonlinear equation is given by

$$
\Phi_{\text {nonlinear }}^{h}(u)=\mathcal{Q}\left(e^{-i \lambda|u|^{2} h} u\right) ;
$$

that is, $\Phi_{\text {nonlinear }}^{h}(u)\left(x_{j}\right)=e^{-i \lambda\left|u\left(x_{j}\right)\right|^{2} h} u\left(x_{j}\right)$ for all $j \in \mathcal{K}$. This is easy to compute in terms of the function values in the collocation points. Note that the fast Fourier transform provides an efficient tool to switch from Fourier coefficients to function values in the collocation points, and vice versa. The computational cost per time step is thus of order $K^{d} \log K^{d}$.

Plane waves in the split-step Fourier method. The split-step Fourier method (4) has plane wave solutions

$$
u_{K}^{n}(x)=\rho e^{i\left(\ell \cdot x-\omega t_{n}\right)} \quad \text { for } u_{K}^{0}(x)=\rho e^{i(\ell \cdot x)}
$$

with $\omega=|\ell|^{2}+\lambda \rho^{2}$ and $\ell \in \mathcal{K}$. In other words, the plane wave solutions $\rho e^{i(\ell \cdot x-\omega t)}$ (2) of the nonlinear Schrödinger equation (1) are integrated exactly by the splitstep Fourier method if $\ell \in \mathcal{K}$. It is the stability of these plane wave solutions (7) under perturbations of the initial value that we are interested in.

2.2. Long-time orbital stability. For the study of the stability of plane wave solutions (7), with fixed vector $\ell \in \mathcal{K}$, we impose the following assumptions (with constants that do not depend on the discretization parameters $h$ and $K$ ).

ASSUMPTION 1. We assume that the time step-size $h$ and the spatial discretization parameter $K$ fulfill together with $\rho \geqslant 0$ (which will be chosen later as the $L_{2}$-norm of the initial value)

$$
\left(\cos (n(j) h)-h \lambda \rho^{2} \sin (n(j) h)\right)^{2} \leqslant 1-c_{1} h^{2} \text { for all } j \in \mathcal{Z}:=\mathcal{K} \backslash\{0\}
$$

with a positive constant $c_{1}$, where

$$
n(j)=\frac{1}{2}|\ell+j \bmod 2 K|^{2}+\frac{1}{2}|\ell-j \bmod 2 K|^{2}-|\ell|^{2} .
$$

By $\ell+j \bmod 2 K$, we denote the element of $\mathcal{K}$ that is entrywise congruent modulo $2 K$ to $\ell+j$, and similarly for $\ell-j \bmod 2 K$. 
Assumption 1 ensures that the frequencies

$$
\begin{aligned}
\omega_{j}= & \frac{1}{2}|\ell+j \bmod 2 K|^{2}-\frac{1}{2}|\ell-j \bmod 2 K|^{2} \\
& +\frac{\arccos \left(\cos (n(j) h)-h \lambda \rho^{2} \sin (n(j) h)\right)}{h \operatorname{sgn}\left(\sin (n(j) h)+h \lambda \rho^{2} \cos (n(j) h)\right)}
\end{aligned}
$$

are well defined for all $j \in \mathcal{Z}$. These frequencies show up after a linearization of the split-step Fourier method around a plane wave. This linearization has eigenvalues $e^{-i \omega_{j} h}$; see Section 3.

As a second assumption, we need a nonresonance condition. Ideally we would like to impose this condition directly on the frequencies $\omega_{j}$. For the verification of the nonresonance condition, however, it turns out to be appropriate to consider modifications of these frequencies.

ASSUMPTION 2. We assume that the time step-size $h$, the spatial discretization parameter $K$, and $\rho \geqslant 0$ are chosen such that there exist modified frequencies $\varpi_{j}$, $j \in \mathcal{Z}$, with the following properties for some $N \geqslant 2$.

(i) The modified frequencies are close to the frequencies $\omega_{j}(10)$,

$$
\left|\varpi_{j}-\omega_{j}\right| \leqslant \widehat{\varepsilon} \quad \text { for all } j \in \mathcal{Z}
$$

with a small parameter $\widehat{\varepsilon}$.

(ii) There exist positive constants $c_{2}, \delta_{2}$, and $s_{2}$ such that the following holds for all vectors $\left(k_{j}\right)_{j \in \mathcal{Z}} \in \mathbb{Z}^{\mathcal{Z}}$ of integers with $0<\sum_{j \in \mathcal{Z}}\left|k_{j}\right| \leqslant N+1$ and with $k_{j} \neq 0$ only if $k_{l}=0$ for all indices $l \neq j$ with $\varpi_{l}=\varpi_{j}$ : if

$$
\delta:=\left|\frac{e^{i\left(\sum_{j \in \mathcal{Z}} k_{j} \varpi_{j}\right) h}-1}{h}\right| \leqslant \delta_{2},
$$

then, for all $l \in \mathcal{Z}$ satisfying $k_{l} \neq 0$,

$$
\frac{|l|^{4}}{\prod_{j \in \mathcal{Z}}|j|^{2\left|k_{j}\right|}} \leqslant c_{2} \delta^{N / s_{2}} .
$$

(iii) Complete resonances among the modified frequencies, that is, $h \sum_{j \in \mathcal{Z}} k_{j} \varpi_{j} \in 2 \pi \mathbb{Z}$ for a vector $\left(k_{j}\right)_{j \in \mathcal{Z}}$ of integers with $\sum_{j \in \mathcal{Z}}\left|k_{j}\right| \leqslant N+1$, can only occur if

$$
\sum_{\substack{j \in \mathcal{Z} \\ n(j)=m}} k_{j}=0 \quad \text { for all } m \in \mathbb{Z} .
$$


Under these assumptions, we will prove the following main result. Here, for a trigonometric polynomial $u(x)=\sum_{j \in \mathcal{K}} u_{j} e^{i(j \cdot x)}$, we denote by

$$
\|u\|_{s}^{2}=\left|u_{0}\right|^{2}+\sum_{j \in \mathcal{K}}|j|^{2 s}\left|u_{j}\right|^{2}
$$

its Sobolev $H^{s}$-norm. We further denote by

$$
\mathcal{F}_{\neg \ell}(u)=\mathcal{Q}\left(e^{-i(\ell \cdot x)} u-u_{\ell}\right)=\sum_{0 \neq j \in \mathcal{K}} u_{j+\ell \bmod 2 K} e^{i(j \cdot x)}
$$

the same function with the $\ell$ th Fourier coefficient set to zero, followed by a shift of Fourier coefficients by $\ell$ and a trigonometric interpolation. Note that $\left\|\mathcal{F}_{\neg \ell}(u)\right\|_{s}$ measures the size of those Fourier coefficients whose subscript differs from $\ell$ modulo $2 K$.

THEOREM 1. Fix an index $\ell \in \mathcal{K}$, an integer $N \geqslant 2$, and positive numbers $c_{1}, c_{2}$, $s_{2}, \delta_{2}$, and $\rho_{1}$. There exist $s_{0}$ and $C$ such that for every $s \geqslant s_{0}$ there exists $\varepsilon_{0}>0$ such that the following holds. If the time step-size $h$, the spatial discretization parameter $K$, and $\rho \leqslant \rho_{1}$ fulfill Assumptions 1 and 2 with some $\widehat{\varepsilon} \leqslant \varepsilon_{0}$ (and with the prescribed constants $c_{1}, c_{2}, s_{2}, \delta_{2}$ ), then, for every initial value $u_{K}^{0}$ with

$$
\left\|u_{K}^{0}\right\|_{0}=\rho \text { and }\left\|\mathcal{F}_{\neg \ell}\left(u_{K}^{0}\right)\right\|_{s} \leqslant \varepsilon \leqslant \varepsilon_{0},
$$

we have the long-time stability estimate

$$
\left\|\mathcal{F}_{\neg \ell}\left(u_{K}^{n}\right)\right\|_{s} \leqslant C \varepsilon \quad \text { for } 0 \leqslant t_{n}=n h \leqslant \max (\varepsilon, \widehat{\varepsilon})^{-N / 2} .
$$

The proof of this theorem will be given in Sections 3-4. Theorem 1 states that, under suitable assumptions, initial values that are close to a plane wave lead to numerical solutions that remain close to a plane wave for a long time; that is, the numerical solution is concentrated in a single Fourier mode over long times. The closeness is measured by the Sobolev $H^{s}$-norm of $\mathcal{F}_{\neg \ell}(u)$. This implies long-time orbital stability in $H^{s}$; that is, the numerical solution stays close to the orbit (7); see [8, Section 3.4].

The bounds $s_{0}, C$, and $\varepsilon_{0}$ are independent of the discretization parameters $h$ and $K$ subject to Assumptions 1 and 2 and of the small parameters $\varepsilon$ and $\widehat{\varepsilon}$. In more detail, the proof of Theorem 1 shows that $s_{0}$ depends only on $d$ and $s_{2} ; C$ depends only on $c_{1}$ and $\rho_{1}$; and $\varepsilon_{0}$ depends on $c_{1}, c_{2}, d, \ell, N, s, s_{2}, \delta_{2}$, and $\rho_{1}$.

REMARK 2. The conclusion of Theorem 1 equally holds if the (Lie-Trotter) splitting (4a) is replaced by its symmetric version, the Strang splitting

$$
u_{K}^{n+1}=\Phi_{\text {linear }}^{h / 2} \circ \Phi_{\text {nonlinear }}^{h} \circ \Phi_{\text {linear }}^{h / 2}\left(u_{K}^{n}\right) .
$$


In fact, both numerical schemes differ only by half a time step with the linear flow at the beginning and at the end of the interval of integration. This does not affect the long-time stability. The same remark applies to the other version of the Strang splitting,

$$
u_{K}^{n+1}=\Phi_{\text {nonlinear }}^{h / 2} \circ \Phi_{\text {linear }}^{h} \circ \Phi_{\text {nonlinear }}^{h / 2}\left(u_{K}^{n}\right) .
$$

2.3. Discussion of the assumptions. Assumptions 1 and 2 for Theorem 1 exclude two different types of (potential) instabilities that show up on different time scales. Assumption 1, which is derived in [25, Section 5] and [22] with a slightly different meaning of $\rho$, ensures that (numerical) plane wave solutions (7) are linearly stable. This means that all eigenvalues of the linearization of the numerical scheme (4) around a plane wave (7) are of modulus one. Eigenvalues of modulus larger than one would lead to an instability right from the start. In contrast, the nonresonance condition of Assumption 2 on the frequencies is crucial for the proof of our long-time result. Indeed, the longer the time interval under consideration is, the more the nonlinear interaction becomes relevant, possibly leading to resonance phenomena if the frequencies are resonant or close to resonant.

A nonresonance condition as stated in Assumption 2 is typically required in a long-time analysis of Hamiltonian partial differential equations and their numerical discretizations; see for example [5, 9-11, 13] for uses and discussions of similar conditions. Note, however, that we do not (and cannot) impose this nonresonance condition on the completely resonant frequencies of the nonlinear Schrödinger equation (1) and its discretization by the split-step Fourier method, but only on the frequencies $\omega_{j}$ of (10) for the linearization around a plane wave.

In Section 5, we will prove the following theorem on a sufficient (though not necessary) condition under which Assumptions 1 and 2 hold in the case of a constant plane wave $(\ell=0)$ for many values of $\rho=\left\|u_{K}^{0}\right\|_{0}$ and $h$.

THEOREM 3. Let $\ell=0$, and fix $\rho_{0}>0$ with

$$
1+2 \lambda \rho_{0}^{2}>0
$$

$h_{0}>0$, and $N \geqslant 2$. Then we have the following result.

For every $\gamma>0$ there exists a subset $\mathcal{P}(\gamma)$ of $\left[0, \rho_{0}\right] \times\left[0, h_{0}\right]$ of Lebesgue measure $|\mathcal{P}(\gamma)| \geqslant \rho_{0} h_{0}-\gamma$ such that Assumptions 1 and 2 hold for all $(\rho, h) \in \mathcal{P}(\gamma)$ and all $K$ that satisfy the restriction

$$
d h K^{2}+2 h \rho_{0}^{2} \leqslant \frac{\pi}{N+1}
$$

with small parameter $\widehat{\varepsilon}=C_{2} h^{2}$ and constants $c_{1}=c_{1}\left(\rho_{0}\right), C_{2}=C_{2}\left(\rho_{0}\right), c_{2}=$ $c_{2}\left(h_{0}, N, \gamma, \rho_{0}\right), \delta_{2}=1$, and $s_{2}=5^{4} N^{5}$. 
Theorem 1 together with Theorem 3 is the discrete counterpart of [8, Theorem 1.1]: for $\ell=0$, the long-time orbital stability of plane waves proven there for the exact solution transfers to the numerical discretization provided that the stepsize restriction (12) is fulfilled and that $\rho=\left\|u_{K}^{0}\right\|_{0}$ and $h$ belong to a large set. In comparison with the result [8, Theorem 1.1] for the exact solution, our discrete counterpart is valid on a time interval of length $\max \left(\varepsilon, h^{2}\right)^{-N / 2}$ instead of $\varepsilon^{-N / 2}$, and the value of $N$ is restricted by the step-size restriction (12). These changes are due to the nonresonance condition that is much more involved for the numerical frequencies than for the analytical frequencies.

Let us finally comment on condition (11) in Theorem 3. This condition ensures linear stability of (analytical) plane wave solutions (2) to the nonlinear Schrödinger equation (1); that is, that all eigenvalues of the linearization of the nonlinear Schrödinger equation around a plane wave (2) are real valued $[\mathbf{1 , 8}$, 25]. Theorem 3 states in particular that this implies linear stability of (numerical) plane wave solutions ((8) in Assumption 1) under the step-size restriction (12). Actually, weaker step-size restrictions that yield linear stability are discussed in detail in [25, Section 5], but (12) is sufficient for our long-time result because it allows us to verify Assumption 2. On the other hand, (8) reduces to (11) (with $\rho_{0}=\rho$ ) in the limit $h \rightarrow 0$ for fixed $K$.

For nonzero but small $\ell$, the condition of linear stability in Assumption 1 can still be expected to hold under a step-size restriction similar to (12). In this case, the frequencies $\omega_{j}$ differ from those for $\ell=0$ only for large $j$ (we have $n(j)=$ $|j|^{2}$ and $|\ell+j \bmod 2 K|=|\ell-j \bmod 2 K|$ for all $j$ that are not large, and we have $c|j|^{2} \leqslant n(j) \leqslant C|j|^{2}$ for large $\left.j\right)$. This property can also be used to argue that the nonresonance condition of Assumption 2 can be expected to hold for nonzero but small $\ell$; see Remark 23 in Section 5. In one dimension $(d=1)$, the linear stability of the split-step Fourier method for $\ell \neq 0$ has been recently analyzed in detail by Lakoba [22].

2.4. Numerical experiments. We present numerical experiments which illustrate Theorem 1 in situations that are not covered by Theorem 3. They show in particular that the conditions of Theorem 3 are not necessary for the assumptions and conclusions of Theorem 1 to hold.

Throughout, we let $\lambda=-1$ and $\rho^{2}=0.4$ such that $1+2 \lambda \rho^{2}>0$, and hence we have linear stability of plane waves in the exact solution. We consider the nonlinear Schrödinger equation in dimension one $(d=1)$ with an initial value that is chosen randomly such that, for $\ell=0, s=5$, and $\varepsilon=0.01$,

$$
\left\|u_{K}^{0}\right\|_{0}=\rho \quad \text { and }\left\|\mathcal{F}_{\neg \ell}\left(u_{K}^{0}\right)\right\|_{s}=\varepsilon ;
$$

that is, the initial value is, in the $H^{5}$-norm, up to 0.01 close to the constant plane wave $\rho$. 

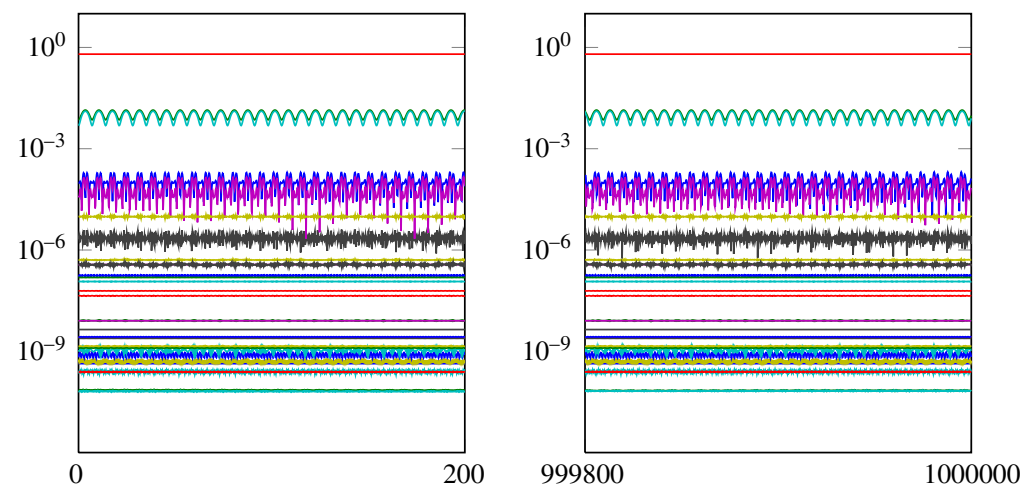

Figure 1. Evolution of the absolute values of the Fourier coefficients for $h=0.04$.

For the numerical discretization with the split-step Fourier method we use $2^{5}$ points for the Fourier collocation in space $\left(K=2^{4}\right)$, and we consider three different step-sizes for the discretization in time: the step-size $h=0.04$ that does not fulfill the step-size restriction (12) of Theorem 3, and the slightly larger stepsizes $h=0.042$ and $h=0.044$.

We have checked numerically for $h=0.04$ and $h=0.044$ that Assumptions 1 and 2 of Theorem 1 are fulfilled for $N \leqslant 5$ with $c_{1}=0.2, \widehat{\varepsilon}=0, c_{2}=8, \delta_{2}=0.1$, and $s_{2}=5 \mathrm{~N}$ for $h=0.04$ and $s_{2}=8 \mathrm{~N} / 5$ for $h=0.044$. Note, however, that the step-size restriction (12) of Theorem 3 is not fulfilled. For the evolution shown in Figure 1, we computed with step-size $h=0.04$ on a long time interval $t \leqslant 10^{6}$ and plotted the absolute values of the Fourier coefficients on two subintervals of length 200. The same was done for Figure 2 with the step-size $h=0.044$. As stated in Theorem 1, we observe in both cases that the solution stays concentrated in the $\ell$ th Fourier mode over long times.

For the intermediate step size $h=0.042$, however, Assumption 1 of Theorem 1 is not fulfilled. In Figure 3 we again plotted the absolute values of the Fourier coefficients of the numerical solution, and we clearly observe an instability.

\section{Reductions and transformations}

From now on we omit the index $K$ of the numerical solution $u_{K}^{n}, n=0$, $1,2, \ldots$ Instead, we denote by $u_{j}^{n}$ the $j$ th Fourier coefficient of $u^{n}: u^{n}(x)=$ $\sum_{j \in \mathcal{K}} u_{j}^{n} e^{i(j \cdot x)}$. We work with the numerical scheme (4a) in terms of these Fourier coefficients, which takes the form (see (5) and (6)) 

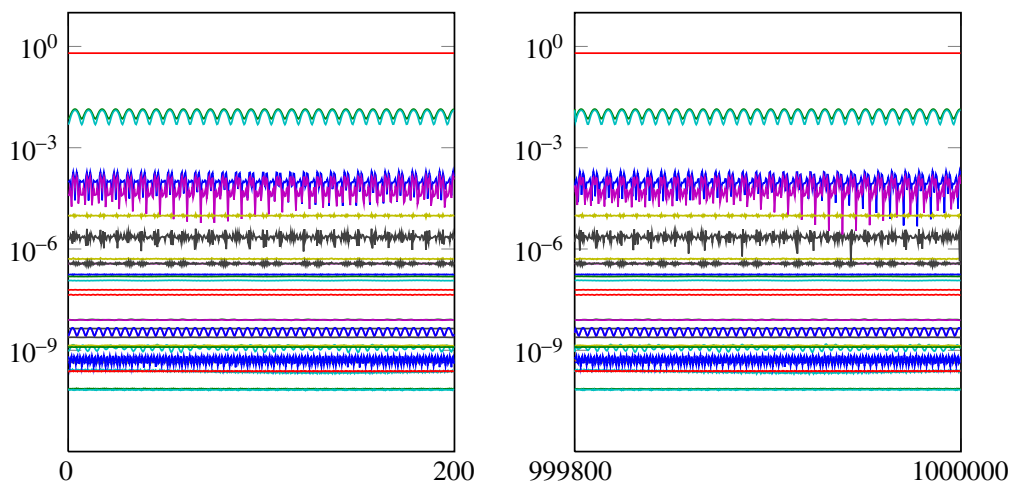

Figure 2. Evolution of the absolute values of the Fourier coefficients for $h=0.044$.

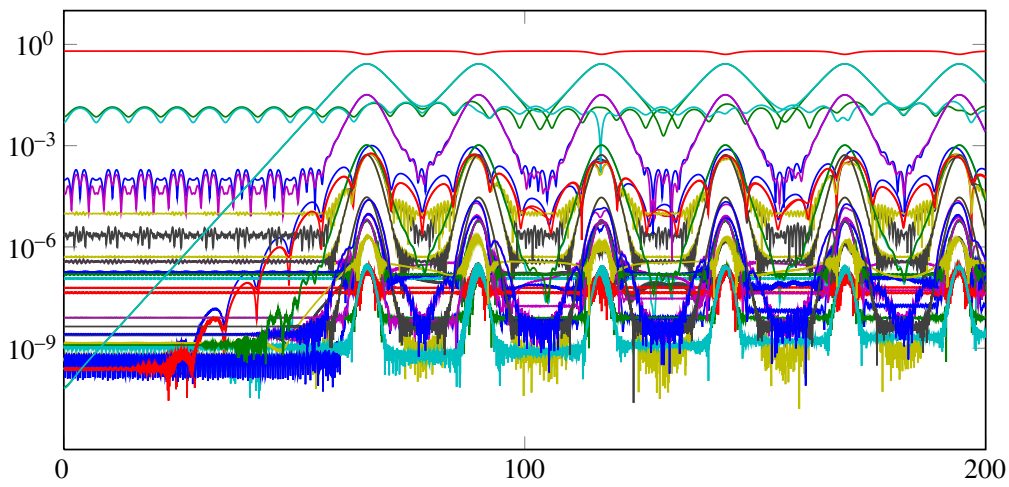

Figure 3. Evolution of the absolute values of the Fourier coefficients for $h=0.042$.

$$
u_{j}^{n+1}=e^{-i|j|^{2} h} \sum_{m=0}^{\infty} \frac{(-i h \lambda)^{m}}{m !} \sum_{\substack{k^{1}+\cdots+k^{m+1} \\-l^{1}+\cdots, l^{m} \equiv j \bmod 2 K}} u_{k^{1}}^{n} \cdots u_{k^{m+1}}^{n} \bar{u}_{l^{1}}^{n} \cdots \bar{u}_{l^{m}}^{n} .
$$

The goal of this section is to eliminate the $\ell$ th Fourier mode, which is not small, from $u^{n}$. To this end we apply similar reductions and transformations to those for the exact solution in [8, Section 2], which can be summarized as follows.

(1) Transformation $u \leftrightarrow v$ with $u=\left(u_{j}\right)_{j \in \mathcal{K}}, v=\left(v_{j}\right)_{j \in \mathcal{K}}$ : shift to the case $\ell=0$; see Section 3.1.

(2) Transformation $v \leftrightarrow(a, \theta, w)$ with $a, \theta \in \mathbb{R}$ and $w=\left(w_{j}\right)_{j \in \mathcal{K} \backslash\{0\}}$ : introduction of polar coordinates $(a, \theta)$ for $v_{0}$ and rotations $w_{j}$ of $v_{j}$; see Section 3.2. 
(3) Reduction $(a, \theta, w) \leftrightarrow w$ : elimination of $a$ and $\theta$ using conservation of mass and gauge invariance; see Section 3.2.

(4) Transformation $w \leftrightarrow \xi$ with $\xi=\left(\xi_{j}\right)_{j \in \mathcal{K} \backslash\{0\}}$ : diagonalization of the linear part; see Sections 3.3-3.4.

These transformations and reductions are applied directly to the numerical scheme in the form (13). In Section 3.5, we consider them from a different perspective, namely from the perspective of the differential equations that form the two steps of the splitting integrator (4a). Both perspectives will be important in the following section, Section 4.

3.1. Shift to the case $\ell=0$. We introduce new variables

$$
v_{j}=u_{\ell+j \bmod 2 K} \quad \text { for } j \in \mathcal{K} .
$$

The numerical scheme (13) in the new variables $v^{n}$ becomes

$$
v_{j}^{n+1}=e^{-i|\ell+j \bmod 2 K|^{2} h} \sum_{m=0}^{\infty} \frac{(-i h \lambda)^{m}}{m !} \sum_{\substack{k^{1}+\ldots+k^{m+1} \\-l^{1} \ldots-l^{m} \equiv j \bmod 2 K}} v_{k^{1}}^{n} \cdots v_{k^{m+1}}^{n} \bar{v}_{l^{1}}^{n} \cdots \bar{v}_{l^{m}}^{n} .
$$

3.2. Elimination of the zero mode. We introduce polar coordinates $(a, \theta)$ for $v_{0}$,

$$
v_{0}=a e^{i \theta} \quad \text { with } a=\left|v_{0}\right|,
$$

and new variables $w_{j}, 0 \neq j \in \mathcal{K}$, by

$$
v_{j}=w_{j} e^{i \theta} \quad \text { for } j \in \mathcal{Z}=\mathcal{K} \backslash\{0\} .
$$

In these new variables $(a, \theta, w)$ with $w=\left(w_{j}\right)_{j \in \mathcal{Z}}$, the numerical scheme (14) becomes

$$
\begin{aligned}
w_{j}^{n+1}= & e^{-i|\ell+j \bmod 2 K|^{2} h} e^{i\left(\theta^{n}-\theta^{n+1}\right)} \sum_{m=0}^{\infty} \frac{(-i h \lambda)^{m}}{m !} \\
& \times \sum_{\substack{k^{1}+\ldots+k^{m+1} \\
-l^{1}-\ldots-l^{m} \equiv j \bmod 2 K}} w_{k^{1}}^{n} \cdots w_{k^{m+1}}^{n} \bar{w}_{l^{1}}^{n} \cdots \bar{w}_{l^{m}}^{n},
\end{aligned}
$$

where we use the convention $w_{0}^{n}=\bar{w}_{0}^{n}=a^{n}$.

Now, we eliminate $a$ and $\theta$ from (16). For the elimination of $a$ we observe that the split-step Fourier method (13) conserves mass, 


$$
\left\|u^{n+1}\right\|_{0}=\sum_{j \in \mathcal{K}}\left|u_{j}^{n+1}\right|^{2}=\sum_{j \in \mathcal{K}}\left|u_{j}^{n}\right|^{2}=\left\|u^{n}\right\|_{0}
$$

a fact that can be easily derived from the representations (5) and (6) of the flows composing the numerical scheme and the discrete Parseval identity $\sum_{j \in \mathcal{K}}\left|u_{j}^{n}\right|^{2}=$ $(2 K)^{-d} \sum_{k \in \mathcal{K}}\left|u^{n}\left(x_{k}\right)\right|^{2}$. The conservation of mass allows us to express $a^{n}$ in terms of $w_{j}^{n}, j \in \mathcal{Z}$, and $\rho=\left\|u^{0}\right\|_{0}$ :

$$
a^{n}=\left(\rho^{2}-\sum_{j \in \mathcal{Z}}\left|w_{j}^{n}\right|^{2}\right)^{1 / 2}
$$

Also, the factor $e^{i\left(\theta^{n}-\theta^{n+1}\right)}$ in (16) can be expressed in terms of $w_{j}$ using (16) for $j=0$ :

$$
e^{i\left(\theta^{n}-\theta^{n+1}\right)}=\frac{e^{i|\ell|^{2} h}}{w_{0}^{n+1}} \sum_{m=0}^{\infty} \frac{(i h \lambda)^{m}}{m !} \sum_{\substack{k^{1}+\ldots+k^{m+1} \\-l^{1}-\ldots-l^{m} \equiv 0 \bmod 2 K}} \bar{w}_{k^{1}}^{n} \cdots \bar{w}_{k^{m+1}}^{n} w_{l^{1}}^{n} \cdots w_{l^{m}}^{n} .
$$

Hence, $a=w_{0}=\bar{w}_{0}$ and $e^{i \theta}$ are determined by $w_{j}, j \in \mathcal{Z}$. The numerical scheme (16) is therefore completely described by the reduced set of variables $w=\left(w_{j}\right)_{j \in \mathcal{Z}}$.

Now, we can replace $w_{0}^{n}=a^{n}$ in (16) and (18) by (17). Furthermore, we can use (17) with $n+1$ instead of $n$ and $\left|w_{j}^{n+1}\right|^{2}$ replaced by (16) to replace $w_{0}^{n+1}$ in (18). For sufficiently small $w$ this leads, after a Taylor expansion of $\left(\rho^{2}-\cdots\right)^{ \pm 1 / 2}$, to an equation for $w^{n+1}$ of the following form (with right-hand side depending only on $\left.w^{n}\right)$ :

$$
\begin{aligned}
w_{j}^{n+1}= & e^{-i\left(|\ell+j \bmod 2 K|^{2}-|\ell|^{2}\right) h}\left(\left(1-i h \lambda \rho^{2}\right) w_{j}^{n}-i h \lambda \rho^{2} \bar{w}_{-j}^{n}\right. \\
& \left.+\sum_{m+m^{\prime}=2}^{\infty} \sum_{\substack{k^{1}+\cdots+k^{m} \\
-l^{1}-\ldots-l^{m^{\prime}} \equiv j \bmod 2 K}}^{\infty} h \widetilde{Q}_{j, k, l} w_{k^{1}}^{n} \cdots w_{k^{m}}^{n} \bar{w}_{l^{1}}^{n} \cdots \bar{w}_{l^{m^{\prime}}}^{n}\right) .
\end{aligned}
$$

The subscripts here and in the following all belong to the reduced set $\mathcal{Z}$.

3.3. Linear stability and numerical frequencies. The linear part in Equation (19) couples $w_{j}$ to $\bar{w}_{-j}$. This leads us to consider the equation for $w_{j}$ together with the one for $\bar{w}_{-j}$,

$$
\left(\begin{array}{c}
w_{j}^{n+1} \\
\bar{w}_{-j}^{n+1}
\end{array}\right)=e^{-i\left(|\ell+j \bmod 2 K|^{2} / 2-|\ell-j \bmod 2 K|^{2} / 2\right) h} A_{j}\left(\begin{array}{c}
w_{j}^{n} \\
\bar{w}_{-j}^{n}
\end{array}\right)+\text { higher-order terms }
$$


with the matrix

$$
A_{j}=\left(\frac{\alpha_{j}}{\beta_{j}} \frac{\beta_{j}}{\alpha_{j}}\right)
$$

where

$$
\begin{gathered}
\alpha_{j}=\left(1-i h \lambda \rho^{2}\right) e^{-i\left(|\ell+j \bmod 2 K|^{2} / 2+|\ell-j \bmod 2 K|^{2} / 2-|\ell|^{2}\right) h}, \\
\beta_{j}=-i h \lambda \rho^{2} e^{-i\left(|\ell+j \bmod 2 K|^{2} / 2+|\ell-j \bmod 2 K|^{2} / 2-|\ell|^{2}\right) h} .
\end{gathered}
$$

This matrix has $\left|\alpha_{j}\right|^{2}-\left|\beta_{j}\right|^{2}=1$, and its eigenvalues are

$$
\lambda_{j}^{ \pm}=\operatorname{Re}\left(\alpha_{j}\right) \pm i \operatorname{sgn}\left(\operatorname{Im}\left(\alpha_{j}\right)\right) \sqrt{1-\operatorname{Re}\left(\alpha_{j}\right)^{2}} .
$$

The reason for including the sign $\operatorname{sgn}\left(\operatorname{Im}\left(\alpha_{j}\right)\right)$ in the definition of the eigenvalues $\lambda_{j}^{ \pm}$will become clear in the following section, Section 3.4.

Assumption 1 ensures that $\operatorname{Re}\left(\alpha_{j}\right)^{2} \leqslant 1$, and hence the eigenvalues $\lambda_{j}^{ \pm}$of $A$ are of modulus one. We have

$$
e^{-i\left(|\ell+j \bmod 2 K|^{2} / 2-|\ell-j \bmod 2 K|^{2} / 2\right) h} \lambda_{j}^{ \pm}=e^{\mp i \omega_{j} h}
$$

with the numerical frequencies $\omega_{j}$ from (10),

$$
\omega_{j}=\frac{1}{2}|\ell+j \bmod 2 K|^{2}-\frac{1}{2}|\ell-j \bmod 2 K|^{2}+\frac{\arccos \left(\operatorname{Re}\left(\alpha_{j}\right)\right)}{-h \operatorname{sgn}\left(\operatorname{Im}\left(\alpha_{j}\right)\right)},
$$

where the branch of arccos with values in $[0, \pi]$ is used. Note that eigenvalues of $A_{j}$ of modulus greater than one would lead to a growth of the corresponding modes in the linearization of (19). Assumption 1 excludes this scenario and thus ensures linear stability of the split-step Fourier method.

3.4. Diagonalization of the linear part. We introduce new variables $\xi_{j}$ that diagonalize the linear part of (19):

$$
\left(\frac{\xi_{j}}{\xi_{-j}}\right)=S_{j}\left(\begin{array}{c}
w_{j} \\
\bar{w}_{-j}
\end{array}\right)
$$

where, with the notation of the previous subsection,

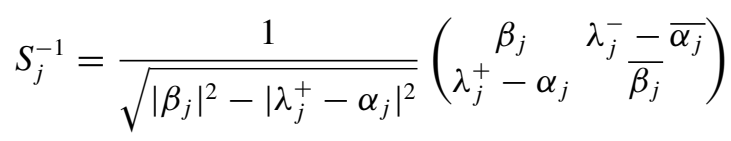

such that

$$
e^{-i\left(|\ell+j \bmod 2 K|^{2} / 2-|\ell-j \bmod 2 K|^{2} / 2\right) h} S_{j} A_{j} S_{j}^{-1}=\left(\begin{array}{cc}
e^{-i \omega_{j} h} & 0 \\
0 & e^{i \omega_{j} h}
\end{array}\right) .
$$


Note that

$$
\left|\beta_{j}\right|^{2}-\left|\lambda_{j}^{+}-\alpha_{j}\right|^{2}=2 \sqrt{1-\operatorname{Re}\left(\alpha_{j}\right)^{2}}\left(\left|\operatorname{Im}\left(\alpha_{j}\right)\right|-\sqrt{1-\operatorname{Re}\left(\alpha_{j}\right)^{2}}\right)>0,
$$

and hence this change of variables, which defines $\xi_{j}$ and $\bar{\xi}_{j}$, is well defined because of the structure of $S_{j}$ (this is the reason for including the sign of $\operatorname{Im}\left(\alpha_{j}\right)$ in the definition of the numerical frequencies). Moreover, it is symplectic, since $\operatorname{det}\left(S_{j}\right)=1$. With this change of variables, (19) is transformed to

$$
\xi_{j}^{n+1}=e^{-i \omega_{j} h} \xi_{j}^{n}+\sum_{m+m^{\prime}=2}^{\infty} \sum_{\substack{k^{1}+\ldots+k^{m} \\-l^{1}-\cdots-l^{m^{\prime}} \equiv j \bmod 2 K}}^{\infty} h Q_{j, k, l} \xi_{k^{1}}^{n} \cdots \xi_{k^{m}}^{n} \bar{\xi}_{l^{1}}^{n} \cdots \bar{\xi}_{l^{m^{\prime}}}^{n} .
$$

\subsection{The splitting structure of the numerical scheme in the new variables.} Recall that, in the original variables $u$,

$$
u^{n+1}=\Phi_{\text {linear }}^{h} \circ \Phi_{\text {nonlinear }}^{h}\left(u^{n}\right) ;
$$

see Equation (4a). Here, $\Phi_{\text {linear }}^{h}=\Phi_{\breve{H}_{0}}^{h}$ is the flow at time $h$ of the Hamiltonian differential equation with Hamiltonian function $\breve{H}_{0}$,

$$
i \dot{u}_{j}=|j|^{2} u_{j}=\frac{\partial \breve{H}_{0}}{\partial \bar{u}_{j}}(u, \bar{u}) \quad \text { with } \breve{H}_{0}(u, \bar{u})=\sum_{j}|j|^{2} u_{j} \bar{u}_{j} .
$$

Correspondingly, $\Phi_{\text {nonlinear }}^{h}=\Phi_{\breve{P}}^{h}$ is the flow at time $h$ of the Hamiltonian differential equation with Hamiltonian function $\breve{P}$,

$$
i \dot{u}_{j}=\frac{\partial \breve{P}}{\partial \bar{u}_{j}}(u, \bar{u}) \quad \text { with } \breve{P}(u, \bar{u})=\frac{\lambda}{2} \sum_{j^{1}+j^{2}-j^{3}-j^{4} \equiv 0 \bmod 2 K} u_{j^{1}} u_{j^{2}} \bar{u}_{j^{3}} \bar{u}_{j^{4}} .
$$

Now, we consider the transformations $u \leftrightarrow v \leftrightarrow(a, \theta, w) \leftrightarrow w \leftrightarrow \xi$ from the previous subsections on the level of these differential equations (instead of their flows, as we have done in the previous subsections).

Shift $u \leftrightarrow v$. After the change of variables $u \leftrightarrow v$ described in Section 3.1 (leading to the numerical scheme (14)), the splitting scheme becomes

$$
v^{n+1}=\Phi_{\breve{H}_{0}}^{h} \circ \Phi_{\check{P}}^{h}\left(v^{n}\right),
$$

with the Hamiltonian functions

$$
\check{H}_{0}(v, \bar{v})=\sum_{j}|\ell+j \bmod 2 K|^{2} v_{j} \bar{v}_{j}
$$


and

$$
\check{P}(v, \bar{v})=\frac{\lambda}{2} \sum_{j^{1}+j^{2}-j^{3}-j^{4} \equiv 0 \bmod 2 K} v_{j^{1}} v_{j^{2}} \bar{v}_{j^{3}} \bar{v}_{j^{4}} .
$$

Transformation $v \leftrightarrow(a, \theta, w)$. In the variables $(a, \theta, w)$ introduced at the beginning of Section 3.2 (leading to the numerical scheme (16)), the flow of the Hamiltonian differential equation with Hamiltonian function $\check{H}_{0}$ has to be replaced by the flow of

$$
i \dot{w}_{j}=\dot{\theta} w_{j}+|\ell+j \bmod 2 K|^{2} w_{j} .
$$

The corresponding equation for $a=w_{0}$ becomes, after taking the real part,

$$
0=\dot{\theta} a+|\ell|^{2} a \text {. }
$$

Correspondingly, the flow of the Hamiltonian differential equation with Hamiltonian function $\check{P}$ has to be replaced by the flow of

$$
i \dot{w}_{j}=\dot{\theta} w_{j}+\frac{\partial \widehat{P}}{\partial \bar{w}_{j}}(a, \theta, w, \bar{w}) \quad \text { with } \widehat{P}(a, \theta, w, \bar{w})=\check{P}(v, \bar{v}) .
$$

Note that the function $\widehat{P}$ is actually independent of $\theta$ (gauge invariance). The equation for $a=w_{0}$ becomes, after taking the real part,

$$
0=\dot{\theta} a+\frac{1}{2} \frac{\partial \widehat{P}}{\partial a}(a, \theta, w, \bar{w}) .
$$

Reduction $(a, \theta, w) \leftrightarrow w$. Solving (24) for $\dot{\theta}$ and inserting this into Equations (23) for $j \neq 0$ shows that (23) becomes, in the reduced set of variables $w$ from Section 3.2 (with the numerical scheme (19)),

$$
i \dot{w}_{j}=\frac{\partial \widetilde{H}_{0}}{\partial \bar{w}_{j}}(w, \bar{w}) \quad \text { with } \tilde{H}_{0}(w, \bar{w})=\sum_{j}\left(|\ell+j \bmod 2 K|^{2}-|\ell|^{2}\right) w_{j} \bar{w}_{j} .
$$

Solving (26) for $\dot{\theta}$ and inserting this into Equations (25) for $j \neq 0$ yields

$$
i \dot{w}_{j}=\frac{\partial \widehat{P}}{\partial \bar{w}_{j}}(a, \theta, w, \bar{w})+\frac{-w_{j}}{2 a} \frac{\partial \widehat{P}}{\partial a}(a, \theta, w, \bar{w}) .
$$

Using

$$
\frac{\partial a}{\partial \bar{w}_{j}}(w, \bar{w})=\frac{-w_{j}}{2 a}
$$

with $a$ given by (17), we see that Equation (25) becomes, in the reduced set of variables $w$ from Section 3.2 (with the numerical scheme (19)), 


$$
i \dot{w}_{j}=\frac{\partial \widetilde{P}}{\partial \bar{w}_{j}}(w, \bar{w}) \quad \text { with } \widetilde{P}(w, \bar{w})=\widehat{P}(a, \theta, w, \bar{w}),
$$

which surprisingly is again of Hamiltonian form. We hence have

$$
w^{n+1}=\Phi_{\widetilde{H}_{0}}^{h} \circ \Phi_{\widetilde{P}}^{h}\left(w^{n}\right) .
$$

The splitting integrator in the reduced set of variables $w$ is still a Hamiltonian splitting, a splitting into two Hamiltonian equations.

Transformation $w \leftrightarrow \xi$. Concerning the final change of variables $w \leftrightarrow \xi$ of Section 3.4 (leading to the numerical scheme (22)), we note first that the matrix $S_{j}$ was chosen in such a way that it is symplectic. We therefore end up with

$$
\xi^{n+1}=\Phi_{H_{0}}^{h} \circ \Phi_{P}^{h}\left(\xi^{n}\right),
$$

with

$$
H_{0}(\xi, \bar{\xi})=\widetilde{H}_{0}(w, \bar{w}) \quad \text { and } \quad P(\xi, \bar{\xi})=\widetilde{P}(w, \bar{w}) .
$$

While it is an obvious observation that the numerical scheme in the new variables $\xi$ is still a splitting scheme, it is highly remarkable that the split equations retain their Hamiltonian structure.

By virtue of the expansion (22), we have a concrete expression for the flow $\Phi_{P}^{h}\left(\xi^{n}\right)=\left(\Phi_{H_{0}}^{h}\right)^{-1}\left(\xi^{n+1}\right)$,

$$
\Phi_{P}^{h}\left(\xi^{n}\right)=S_{j}\left(\begin{array}{cc}
e^{i\left(|\ell+j \bmod 2 K|^{2}-|\ell|^{2}\right) h} & 0 \\
0 & e^{-i\left(|\ell+j \bmod 2 K|^{2}-|\ell|^{2}\right) h}
\end{array}\right) S_{j}^{-1}\left(\begin{array}{c}
\xi_{j}^{n+1} \\
\xi_{-j}^{n+1}
\end{array}\right) .
$$

For later purposes we also introduce an expansion

$$
P(\xi, \bar{\xi})=\sum_{m+m^{\prime}=2}^{\infty} \sum_{k \in \mathcal{Z}^{m}, l \in \mathcal{Z}^{m^{\prime}}} P_{k, l} \xi_{k^{1}} \cdots \xi_{k^{m}} \bar{\xi}_{l^{1}} \cdots \bar{\xi}_{l^{m^{\prime}}}
$$

of the Hamiltonian function $P$.

3.6. Estimates for the transformation and for the transformed equation. We derive some bounds for the change of variables $u \leftrightarrow \xi$ described in Sections 3.1-3.4. We assume throughout that Assumption 1 is fulfilled.

Note that $\left|w_{j}\right|=\left|u_{\ell+j \bmod 2 K}\right|$ for $j \in \mathcal{Z}$, and hence we first consider the last transformation $w \leftrightarrow \xi$ of Section 3.4 described by the matrices $S_{j}$ (20).

LEMMA 4. The absolute values of the entries of the matrices $S_{j}$ and $S_{j}^{-1}$ are bounded by $\sqrt{1+\rho^{2} /\left(2 \sqrt{c_{1}}\right)}$, independently of $j$ and $h$. 
Proof. With the notation of Section 3.3, we have, by (21),

$$
\begin{aligned}
& \left|\frac{\beta_{j}}{\sqrt{\left|\beta_{j}\right|^{2}-\left|\lambda_{j}^{+}-\alpha_{j}\right|^{2}}}\right|^{2}=\frac{\left|\operatorname{Im}\left(\alpha_{j}\right)\right|+\sqrt{1-\operatorname{Re}\left(\alpha_{j}\right)^{2}}}{2 \sqrt{1-\operatorname{Re}\left(\alpha_{j}\right)^{2}}}, \\
& \left|\frac{\lambda_{j}^{+}-\alpha_{j}}{\sqrt{\left|\beta_{j}\right|^{2}-\left|\lambda_{j}^{+}-\alpha_{j}\right|^{2}}}\right|^{2}=\frac{\left|\operatorname{Im}\left(\alpha_{j}\right)\right|-\sqrt{1-\operatorname{Re}\left(\alpha_{j}\right)^{2}}}{2 \sqrt{1-\operatorname{Re}\left(\alpha_{j}\right)^{2}}} .
\end{aligned}
$$

This proves the statement of the lemma, since $\left|\operatorname{Im}\left(\alpha_{j}\right)\right| \leqslant \sqrt{1-\operatorname{Re}\left(\alpha_{j}\right)^{2}}+h \rho^{2}$, and since $\sqrt{1-\operatorname{Re}\left(\alpha_{j}\right)^{2}} \geqslant \sqrt{c_{1}} h$, by Assumption 1 .

Now, we consider the norm

$$
\|\xi\|_{s}=\left(\sum_{j \in \mathcal{Z}}|j|^{2 s}\left|\xi_{j}\right|^{2}\right)^{1 / 2}
$$

that is, $\|\xi\|_{s}$ is the Sobolev $H^{s}$-norm of the function $\sum_{j \in \mathcal{Z}} \xi_{j} e^{i(j \cdot x)}$ as introduced in Section 2.2. The previous lemma, Lemma 4, implies the following result.

LEMMA 5. For the change of variables $u \leftrightarrow \xi$, there exist positive constants $\widehat{c}$ and $\widehat{C}$ depending only on $c_{1}$ and an upper bound of $\rho$ such that

$$
\widehat{c}\|\xi\|_{s} \leqslant\left\|\mathcal{F}_{\neg \ell}(u)\right\|_{s} \leqslant \widehat{C}\|\xi\|_{s} .
$$

In particular, the previous lemma shows that the condition $\left\|\mathcal{F}_{\neg \ell}\left(u^{0}\right)\right\|_{s} \leqslant \varepsilon$ of Theorem 1 becomes, in the new variables $\xi$,

$$
\left\|\xi^{0}\right\|_{s} \leqslant \widehat{c}^{-1} \varepsilon
$$

We finally collect some estimates for the nonlinearity in the numerical scheme written in the new variables $\xi$ as given by (22).

LEMMA 6. The nonlinearity given by the coefficients $Q_{j, k, l}$ in (22) satisfies, for $s>d / 2$,

$$
\begin{aligned}
& \left(\sum_{j \in \mathcal{Z}}|j|^{2 s}\left(\sum_{\substack{k^{1}+\ldots+k^{m} \\
-l^{1}-\cdots-l^{m^{\prime}} \equiv j \bmod 2 K}}\left|Q_{j, k, l} \eta_{k^{1}}^{1} \cdots \eta_{k^{m}}^{m} \eta_{l^{1}}^{m+1} \cdots \eta_{l^{m^{\prime}}}^{m+m^{\prime}}\right|\right)^{2}\right)^{1 / 2} \\
& \leqslant C_{m, m^{\prime}, s}\left\|\eta^{1}\right\|_{s} \cdots\left\|\eta^{m+m^{\prime}}\right\|_{s}
\end{aligned}
$$

for vectors $\eta^{1}, \ldots, \eta^{m+m^{\prime}} \in \mathbb{C}^{\mathcal{Z}}$. The constants $C_{m, m^{\prime}, s}$ depend only on $m, m^{\prime}, s$, $c_{1}$, and $\rho$, and satisfy

$$
\sum_{m+m^{\prime}=2}^{\infty} C_{m, m^{\prime}, s} r^{m+m^{\prime}} \leqslant C
$$

for some positive constants $r$ and $C$ depending only on $c_{1}, s$, and $\rho$. 
Proof. (a) By carefully going through the construction of the coefficients $Q_{j, k, l}$ in Sections 3.2 and 3.4, one shows for the coefficients $Q_{j, k, l}$ that there exists a constant $C$ depending on $c_{1}$ and $\rho$ such that

$$
\left|Q_{j, k, l}\right| \leqslant C^{m+m^{\prime}}
$$

for all $j \in \mathcal{Z}$, all $k \in \mathcal{Z}^{m}$, and all $l \in \mathcal{Z}^{m^{\prime}}$.

(b) The first estimate of the lemma follows by applying (31) and the CauchySchwarz inequality, and by using

$$
\sum_{\substack{k^{1}+\ldots+k^{m} \\-l^{1}-\cdots-l^{m^{\prime}} \equiv j \bmod 2 K}}\left(\frac{|j|}{\left|k^{1}\right| \cdots\left|k^{m}\right|\left|l^{1}\right| \cdots\left|l^{m^{\prime}}\right|}\right)^{2 s} \leqslant c^{m+m^{\prime}}
$$

with a constant $c$ depending only on $s>d / 2$. The second estimate of the lemma then follows also from (31).

\section{Modulated Fourier expansions}

In this section, we will prove Theorem 1 using modulated Fourier expansions originally introduced in [15]; see also [17]. Throughout, we will work with the numerical scheme in the new variables $\xi$ introduced in Section 3; see (22).

There are two main steps.

(1) Construction of a short-time approximation of $\xi^{n}$ from (22) by a modulated Fourier expansion in Sections 4.1-4.5.

(2) Almost-invariants of the modulated Fourier expansion that allow us to prove a result on a long time interval in Sections 4.6-4.9.

For the first main step, it is convenient to work with the numerical scheme as given by the composition of flows (22), whereas for the derivation of the almost-invariants it is necessary to switch to the level of the differential equations whose flows compose the numerical scheme (27). We ultimately show that $\left\|\xi^{n}\right\|_{s}$ stays of order $\varepsilon$ for initial values $\xi^{0}$ of order $\varepsilon$. This preservation of smallness and regularity of $\xi^{n}$ is the main ingredient for the final proof of Theorem 1 in Section 4.10.

The proof via modulated Fourier expansions given here uses and combines ideas from several previous proofs using such expansions. The aforementioned idea of switching between the flows and the differential equations is loosely based on $[11,13]$, the construction of the modulated Fourier expansion with an asymptotic expansion is based on $[14,16]$, the idea of using modified 
frequencies $\varpi_{j}$ instead of the original (numerical) frequencies $\omega_{j}$ of $(10)$ for the modulated Fourier expansion is also used in [12], the nonresonance condition in Assumption 2 is used in a similar way to [5], and the use of almost-invariants of the modulated Fourier expansion to prove long-time almost-conservation properties can be traced back to [15].

In the following analysis, the (generic) constants $C, s_{0}$, and $\delta_{0}$ are all independent of the small parameters $\varepsilon$ from (30) and $\widehat{\varepsilon}$ from Assumption 2. The constants $C$ and $\delta_{0}$ will depend on the constants $c_{1}, c_{2}, s_{2}, \delta_{2}$, and $N$ of Assumptions 1 and 2, on $s$ from (30), on an upper bound of $\rho=\left\|u^{0}\right\|_{0}$, on the index $\ell \in \mathcal{K}$ from (7), and on the dimension $d$. The constant $s_{0}$ will depend only on $d$ and $s_{2}$.

4.1. Resonant modulated Fourier expansion. In order to motivate the modulated Fourier expansion we consider here, let us first have a look at (22) in the linear case (all $Q_{j, k, l}=0$ ). In this case, the evolution of the $j$ th mode is given by the multiplication with $e^{-i \omega_{j} t}$. In the presence of the nonlinearity, we seek for an expansion, the modulated Fourier expansion, in terms of products of these exponentials that are multiplied (modulated) by slowly varying coefficients.

There are two pitfalls in the present situation that have to be handled with care. First, it turns out that the frequencies $\omega_{j}$ of (10) are inconvenient when it comes to resonance issues. Therefore we use the modified frequencies $\varpi_{j}$ of Assumption 2 instead, and consider products of the exponentials $e^{-i \varpi_{j} t}$ :

$$
e^{-i(\mathbf{k} \cdot \varpi) t} \quad \text { with } \mathbf{k} \cdot \varpi=\sum_{j \in \mathcal{Z}} k_{j} \varpi_{j}
$$

for vectors of integers $\mathbf{k}=\left(k_{j}\right)_{j \in \mathcal{Z}} \in \mathbb{Z}^{\mathcal{Z}}$ and the vector $\varpi=\left(\varpi_{j}\right)_{j \in \mathcal{Z}}$ of modified frequencies.

Second, the modified frequencies $\varpi_{j}$ of Assumption 2 are by definition exactly resonant; for instance, $\varpi_{j}=\varpi_{l}$ for $|j|=|l|$ in the case $\ell=0$. Hence, we cannot distinguish all products $e^{-i(\mathbf{k} \cdot \boldsymbol{\varpi}) t}$, and we therefore introduce the resonance module

$$
\mathcal{M}=\left\{\mathbf{k} \in \mathbb{Z}^{\mathcal{Z}}: \mathbf{k} \cdot \varpi=0, j(\mathbf{k})=0\right\},
$$

where

$$
j(\mathbf{k})=\sum_{l \in \mathcal{Z}} k_{l} l \bmod 2 K .
$$

The restriction $j(\mathbf{k})=0$ in the definition of the resonance module comes from the fact that the products $e^{-i(\mathbf{k} \cdot \boldsymbol{w}) t}$ are attached to some specific mode $\xi_{j}$, namely the one with $j=j(\mathbf{k})$, as we will see in the following. 
With these preliminaries, we introduce the resonant modulated Fourier expansion

$$
\xi_{j}(t)=\sum_{[\mathbf{k}]} z_{j}^{[\mathbf{k}]}(\delta t) e^{-i(\mathbf{k} \cdot \boldsymbol{\varpi}) t}
$$

Here,

$$
\delta=\max (\varepsilon, \widehat{\varepsilon})^{1 / 2}
$$

is a small parameter, and the sum is over all residue classes $[\mathbf{k}] \in \mathbb{Z}^{\mathcal{Z}} / \mathcal{M}$. The coefficients of the modulated Fourier expansion, the modulation functions $z_{j}^{[\mathbf{k}]}$, are required to be polynomials on a slow time scale $\tau=\delta t$ with $\delta$ from (33) that have all derivatives bounded independently of the small parameters. By a slight abuse of notation, we write in the following $z_{j}^{\mathbf{k}}$ instead of $z_{j}^{[\mathbf{k}]}$ and $\sum_{\mathbf{k}}$ instead of $\sum_{[\mathbf{k}]}$. We also use the notation

$$
\|\mathbf{k}\|=\sum_{j \in \mathcal{Z}}\left|k_{j}\right|
$$

4.2. Modulation equations. Requiring $\xi_{j}\left(t_{n}\right)=\xi_{j}^{n}$ for $n \geqslant 1$ with $\xi_{j}^{n}$ given by (22) yields, after a comparison of the coefficients of $e^{i(\mathbf{k} \cdot \boldsymbol{\sigma}) t}$, modulation equations for the modulation functions $z_{j}^{\mathbf{k}}$ :

$$
\begin{aligned}
z_{j}^{\mathbf{k}}(\tau+\delta h) e^{-i(\mathbf{k} \cdot \boldsymbol{w}) h}= & e^{-i \omega_{j} h} z_{j}^{\mathbf{k}}(\tau)+\sum_{m+m^{\prime}=2}^{\infty} \sum_{\substack{\left.\mathbf{k}^{1}+\ldots+\mathbf{k}^{m} \\
-1^{1} \ldots-m^{m^{\prime}} \in \mathbf{k}\right]}} h Q_{j, k, l} z_{k^{1}}^{\mathbf{k}^{1}}(\tau) \cdots z_{k^{m}}^{\mathbf{k}^{m}}(\tau) \bar{z}_{l^{1}}^{1^{1}}(\tau) \cdots \bar{z}_{l^{m^{\prime}}}^{m^{\prime}}(\tau) . \\
& \times \sum_{k \in \mathcal{Z}^{m}, l \in \mathcal{Z}^{m^{\prime}}} h
\end{aligned}
$$

The condition $\xi_{j}(0)=\xi_{j}^{0}$ yields

$$
\sum_{\mathbf{k}} z_{j}^{\mathbf{k}}(0)=\xi_{j}^{0}
$$

For the approximate solution of the modulation equations (34), it is useful to expand the modulation functions in powers of $\varepsilon$ and $\delta$,

$$
z_{j}^{\mathbf{k}}(\tau)=\sum_{p=0}^{\infty} \varepsilon \delta^{p} z_{j, p}^{\mathbf{k}}(\tau),
$$


with polynomials $z_{j, p}^{\mathbf{k}}$ in $\tau=\delta t$. We call the functions $z_{j, p}^{\mathbf{k}}$ modulation coefficient functions, and set $z_{j, p}^{\mathbf{k}}=0$ for $p<0$. After dividing by $\delta h e^{-i(\mathbf{k} \cdot \varpi) h}$, expanding $z_{j}^{\mathbf{k}}(\tau+\delta h)$ around $\tau$, and (formally) comparing the coefficients of $\varepsilon \delta^{p}$, the modulation equations (34a) become

$$
\begin{aligned}
\frac{1-e^{-i\left(\omega_{j}-\mathbf{k} \cdot \boldsymbol{\varpi}\right) h}}{\delta h} z_{j, p}^{\mathbf{k}}+\dot{z}_{j, p}^{\mathbf{k}}= & -\sum_{r=2}^{\infty} \frac{h^{r-1}}{r !} \frac{d^{r}}{d \tau^{r}} z_{j, p+1-r}^{\mathbf{k}}+\sum_{m+m^{\prime}=2}^{\infty} \\
& \times \sum_{\substack{p_{1}+\cdots+p_{m} \\
+q_{1}+\cdots+q_{m^{\prime}}=p+3-2\left(m+m^{\prime}\right)}} \frac{\varepsilon^{m+m^{\prime}-1}}{\delta^{2 m+2 m^{\prime}-2}} \sum_{\substack{\left.\mathbf{k}^{1}+\cdots+\mathbf{k}^{m} \\
-1^{1}-\ldots-\mathbf{k}^{m^{\prime}} \in \mathbf{k}\right]}} \\
& \times \sum_{\substack{k \in \mathcal{Z}^{m}, l \in \mathcal{Z}^{m^{\prime}}\\
}} e^{i(\mathbf{k} \cdot \boldsymbol{w}) h} Q_{j, k, l} z_{k^{1}, p_{1}}^{\mathbf{k}^{1}} \cdots z_{k^{m}, p_{m}}^{\mathbf{k}^{m}} \bar{z}_{l^{1}, q_{1}}^{\mathbf{l}^{1}} \\
& \cdots \bar{z}_{l^{m^{\prime}}, q_{m^{\prime}}}^{m^{\prime}}
\end{aligned}
$$

Condition (34b) yields

$$
z_{j, p}^{\langle j\rangle}(0)=-\sum_{\mathbf{k} \neq\langle j\rangle} z_{j, p}^{\mathbf{k}}(0)+ \begin{cases}\varepsilon^{-1} \xi_{j}^{0}, & p=0, \\ 0, & p>0,\end{cases}
$$

where $\langle j\rangle$ denotes the $j$ th unit vector in $\mathbb{Z}^{\mathcal{Z}}$.

4.3. Construction of modulation functions. We construct modulation functions $z_{j}^{\mathbf{k}}$ that solve the modulation equations (34) up to a small defect. We work with the asymptotic expansion (35) and consider Equations (36). The crucial observation is that the right-hand side of (36a) depends only on modulation coefficient functions $z_{k, q}^{1}$ with $q<p$. This allows us to solve Equations (36) up to a small defect by the following simple recursion.

Fix $p \geqslant 0$, and assume that we have computed all modulation coefficient functions $z_{j, q}^{\mathbf{k}}$ with $q<p$ (this is true for $p=0$ ). Equation (36a) is then of the form

$$
\alpha z_{j, p}^{\mathbf{k}}+\dot{z}_{j, p}^{\mathbf{k}}=P
$$

with a polynomial $P$. The unique polynomial solution of this equation is given for $\alpha \neq 0$ by

$$
z_{j, p}^{\mathbf{k}}(\tau)=\sum_{m=0}^{\operatorname{deg}(P)}(-1)^{m} \alpha^{-m-1} \frac{d^{m}}{d \tau^{m}} P(\tau) .
$$

We therefore compute $z_{j, p}^{\mathbf{k}}$ for all $j$ and all $\mathbf{k}$ as follows. 
(i) For indices $(j, \mathbf{k})$ with $j \neq j(\mathbf{k})$ or $\|\tilde{\mathbf{k}}\|>p$ for all $\tilde{\mathbf{k}} \in[\mathbf{k}]$, we set

$$
z_{j, p}^{\mathbf{k}}=0 \text {. }
$$

This is consistent with (36a), since the right-hand side of this equation vanishes for these indices by induction (recall that $Q_{j, k, l}=0$ if $j \not \equiv k^{1}+$ $\left.\cdots+k^{m}-l^{1}-\cdots-l^{m^{\prime}} \bmod 2 K\right)$.

(ii) For indices $(j, \mathbf{k})$ with $\left|1-e^{-i\left(\omega_{j}-\mathbf{k} \cdot \varpi\right) h}\right| \geqslant \delta h / 2$ that are not covered by (i), we

$$
\text { compute } z_{j, p}^{\mathbf{k}} \text { from (36a) and (37). }
$$

Indeed, the factor in front of $z_{j, p}^{\mathbf{k}}$ in (36a) is bounded for these indices away from zero, and the comparison of coefficients used to derive (36a) thus makes sense.

(iii) For indices $(j, \mathbf{k}) \neq(j,\langle j\rangle)$ that are neither covered by (i) nor covered by (ii), we set

$$
z_{j, p}^{\mathbf{k}}=0 .
$$

Of course, this introduces a defect which, however, can be controlled using the nonresonance condition of Assumption 2, as we shall see in Section 4.5. For the considered indices $(j, \mathbf{k})$, we have $\left|1-e^{-i\left(\omega_{j}-\mathbf{k} \cdot \boldsymbol{\sigma}\right) h}\right|<\delta h / 2$, and they are in this sense close to a resonance. We therefore call them near-resonant indices in the following.

(iv) Having computed $z_{j, p}^{\mathbf{k}}$ for all $j$ and all $\mathbf{k} \neq\langle j\rangle$ in (i)-(iii), we can

$$
\text { compute } z_{j, p}^{\langle j\rangle}(0) \text { from }(36 b) \text {. }
$$

Moreover, since the factor in front of $z_{j, p}^{\mathbf{k}}$ in (36a) vanishes for $\mathbf{k}=\langle j\rangle$, we can

$$
\text { compute } \dot{z}_{j, p}^{\langle j\rangle} \text { from (36a). }
$$

This allows us to compute the diagonal modulation coefficient functions $z_{j, p}^{\langle j\rangle}$. We stop the above construction (38) of modulation coefficient function $z_{j, p}^{\mathbf{k}}$ after $p=N$,

$$
z_{j, p}^{\mathbf{k}}=0 \text { for } p>N .
$$

It is clear that the construction leads to modulation coefficient functions $z_{j, p}^{\mathbf{k}}$ that are polynomials in $\tau$, of degree bounded by $p$. Moreover, we have

$$
z_{j, 0}^{\mathbf{k}}=0 \quad \text { for } \mathbf{k} \neq\langle j\rangle,
$$

because the right-hand side of (36a) vanishes for $p=0$. 
4.4. Size of the modulation functions. We estimate the modulation coefficient functions constructed in (38). For fixed index $p$, we collect them in the vectors

$$
\mathbf{z}_{p}=\left(z_{j, p}^{\mathbf{k}}\right)_{j \in \mathcal{Z}, \mathbf{k} \in \mathbb{Z}^{\mathcal{Z}}}
$$

We also consider their rescalings

$$
\left(\boldsymbol{\Gamma}^{s-\widehat{s}} \mathbf{z}_{p}\right)_{j}^{\mathbf{k}}:=\left(\Gamma^{\mathbf{k}}\right)^{s-\widehat{s}} \cdot z_{j, p}^{\mathbf{k}} \quad \text { with } \Gamma^{\mathbf{k}}:=\min _{\widetilde{\mathbf{k}} \in[\mathbf{k}]}\left(2^{\|\widetilde{\mathbf{k}}\|} \prod_{l \in \mathcal{Z}}|l|^{\left|\widetilde{k}_{l}\right|}\right)
$$

and with $s \geqslant \widehat{s}:=(d+1) / 2$ such that Lemma 6 is applicable for $s$ and $\widehat{s}$.

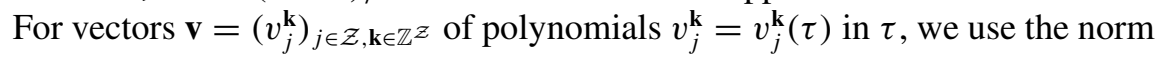

$$
\|\mathbf{v}\|_{s, \tau}=\left\|\left(\sum_{\mathbf{k}}\left|v_{j}^{\mathbf{k}}\right|_{\tau}\right)_{j \in \mathcal{Z}}\right\|_{s}=\left(\sum_{j \in \mathcal{Z}}|j|^{2 s}\left(\sum_{\mathbf{k}}\left|v_{j}^{\mathbf{k}}\right|_{\tau}\right)^{2}\right)^{1 / 2},
$$

where

$$
|v|_{\tau}=\sum_{m=0}^{\infty} \frac{1}{m !}\left|\frac{d^{m}}{d \tau^{m}} v(\tau)\right|
$$

LEMMA 7. The modulation coefficient functions (38) satisfy, on $0 \leqslant \tau \leqslant 1$, for $\delta \leqslant \delta_{0}$ and $s \geqslant \widehat{s}$,

$$
\left\|\mathbf{z}_{p}\right\|_{s, \tau} \leqslant C \quad \text { and } \quad\left\|\boldsymbol{\Gamma}^{s-\widehat{s}} \mathbf{z}_{p}\right\|_{\widehat{s}, \tau} \leqslant C
$$

for all $p$ with constants $C$ and $\delta_{0}$.

Proof. This follows from the recursive construction (38). The property

$$
|v w|_{\tau} \leqslant|v|_{\tau}|w|_{\tau}
$$

together with Lemma 6 yields inductively an estimate of the nonlinearity on the right-hand side of (36a) in the norm $|\|\cdot\||_{s, \tau}$ (note that terms in the nonlinearity with $2\left(m+m^{\prime}\right)>p+3$ vanish, and hence the sum over $m$ and $m^{\prime}$ is finite). Then, the property $|\dot{v}|_{\tau} \leqslant \operatorname{deg}(v)|v|_{\tau}$ allows us to estimate the norm $|\|\cdot\||_{s, \tau}$ for the vector consisting of modulation coefficient functions constructed with (38b). For the remaining nonzero modulation coefficient functions constructed with (38d)(38e), the estimate in the norm $|\|\cdot\||_{s, \tau}$ then follows using the smallness of the initial value (30) and the property $|v|_{\tau} \leqslant|v(0)|+\sup _{0 \leqslant \tau \leqslant \tau}|\dot{v}|_{\tau}$.

For the estimate of the rescaling $\Gamma^{s-\widehat{s}_{p}} \mathbf{z}_{p}$ in the norm $|\|\cdot\||_{\widehat{s}, \tau}$, we can use essentially the same argument. We just have to take into account that

$$
\Gamma^{\mathbf{k}^{1}+\mathbf{k}^{2}} \leqslant \Gamma^{\mathbf{k}^{1}} \Gamma^{\mathbf{k}^{2}}
$$


and that $\Gamma^{\langle j\rangle}=2|j|$. The latter follows from

$$
|j|=|j(\widetilde{\mathbf{k}})| \leqslant \sum_{l \in \mathcal{Z}}\left|\widetilde{k}_{l}\right||l| \leqslant\|\widetilde{\mathbf{k}}\| \prod_{l \in \mathcal{Z}}|l|^{\left|\widetilde{k}_{l}\right|}
$$

for $\tilde{\mathbf{k}} \in[\langle j\rangle]$ and $j \in \mathcal{Z}$.

4.5. Defect and error. The modulation functions constructed in (38) via their modulation coefficient functions (35) are supposed to fulfill the modulation system (34). However, there are two sources of error in their construction. First, we stopped the construction of modulation coefficient functions $z_{j, p}^{\mathbf{k}}$ after $p=N$ (38f). Second, the modulation functions for near-resonant indices $(j, \mathbf{k})$ were set to zero (38c). In other words, the constructed modulation functions satisfy the equations of motion (34a) of the modulation system only up to a defect,

$$
\begin{aligned}
d_{j}^{\mathbf{k}}+e_{j}^{\mathbf{k}}= & -z_{j}^{\mathbf{k}}(\cdot+\delta h) e^{-i(\mathbf{k} \cdot \boldsymbol{\omega}) h}+e^{-i \omega_{j} h} z_{j}^{\mathbf{k}}+\sum_{m+m^{\prime}=2}^{\infty} \sum_{\substack{\mathbf{k}^{1}+\cdots+\mathbf{k}^{m} \\
-\mathbf{1}^{1} \cdots \cdots-\mathbf{l}^{m^{\prime}}=[\mathbf{k}]}} \\
& \times \sum_{k \in \mathcal{Z}^{m}, l \in \mathcal{Z}^{m^{\prime}}} h Q_{j, k, l} z_{k^{1}}^{\mathbf{k}^{1}} \cdots z_{k^{m}}^{\mathbf{k}^{m}} \bar{z}_{l^{1}}^{1^{1}} \cdots \bar{z}_{l^{m^{\prime}}}^{m^{m^{\prime}}},
\end{aligned}
$$

whereas the initial condition (34b) is met exactly. Here, $\mathbf{d}=\left(d_{j}^{\mathbf{k}}\right)_{j \in \mathcal{Z}, \mathbf{k} \in \mathbb{Z}^{z}}$ denotes the defect from the cut-off (38f); that is,

$$
d_{j}^{\mathbf{k}}=\sum_{p=N+1}^{\infty} \varepsilon \delta^{p} h F_{j, p}^{\mathbf{k}} e^{-i(\mathbf{k} \cdot \varpi) h},
$$

where $F_{j, p}^{\mathbf{k}}$ is the right-hand side of (36a). The defect in near-resonant indices that is not yet covered by $\mathbf{d}$ is denoted by $\mathbf{e}=\left(e_{j}^{\mathbf{k}}\right)_{j \in \mathcal{Z}, \mathbf{k} \in \mathbb{Z}^{z}}$; that is, $e_{j}^{\mathbf{k}}$ is different from zero only for near-resonant indices, and in this case

$$
e_{j}^{\mathbf{k}}=\sum_{p=1}^{N} \varepsilon \delta^{p} h F_{j, p}^{\mathbf{k}} e^{-i(\mathbf{k} \cdot \boldsymbol{w}) h} .
$$

(Recall that $F_{j, p}^{\mathbf{k}}=0$ for $p=0$.) Both defects are estimated in the following lemma.

LEMma 8. The defects (42)-(44) satisfy, on $0 \leqslant \tau \leqslant 1$, for $\delta \leqslant \delta_{0}$ and $s \geqslant s_{0}$,

$$
\begin{aligned}
& \|\mathbf{d}\|_{s, \tau} \leqslant C \varepsilon \delta^{N+1} h, \quad\|\mathbf{e}\|_{s, \tau} \leqslant C \varepsilon \delta^{N+1} h, \\
& \left\|\boldsymbol{\Gamma}^{s-\widehat{s}} \mathbf{d}\right\|_{\widehat{s}, \tau} \leqslant C \varepsilon \delta^{N+1} h, \quad\left\|\boldsymbol{\Gamma}^{s-\widehat{s}} \mathbf{e}\right\|_{\widehat{s}, \tau} \leqslant C \varepsilon \delta h
\end{aligned}
$$

with constants $C, s_{0}$ and $\delta_{0}$. 
Proof. (a) For the bound of $\mathbf{d}$, we note that, by Lemmas 6 and 7,

$$
\|\mathbf{d}\|_{s, \tau} \leqslant C \varepsilon \delta^{N+1} h+\varepsilon h \sum_{m+m^{\prime}=2}^{\infty} C_{m, m^{\prime}, s} C^{m+m^{\prime}} \sum_{p=\max \left(N+1,2\left(m+m^{\prime}\right)-3\right)}^{\infty} \delta^{p},
$$

where $C /(N+1)$ denotes the constant of Lemma 7. Splitting the sum over $m$ and $m^{\prime}$ in a part with $m+m^{\prime} \leqslant N+3$ and another part with $m+m^{\prime} \geqslant N+4$ proves the claimed estimate of $\mathbf{d}$ using the second part of Lemma 6 for the sum with $m+m^{\prime} \geqslant N+4$ and sufficiently small $\delta$. The estimates of $\boldsymbol{\Gamma}^{s-\widehat{s}} \mathbf{d}$ and $\boldsymbol{\Gamma}^{s-\widehat{s}} \mathbf{e}$ follow similarly.

(b) Concerning the defect $\mathbf{e}$ in near-resonant indices, we note that, for those indices $(j, \mathbf{k})$,

$$
\begin{aligned}
\left|e^{-i\left(\varpi_{j}-\mathbf{k} \cdot \varpi\right) h}-1\right| & \leqslant\left|e^{-i \varpi_{j} h}-e^{-i \omega_{j} h}\right|+\left|e^{-i\left(\omega_{j}-\mathbf{k} \cdot \varpi\right) h}-1\right| \\
& <2\left|\sin \left(\left(\varpi_{j}-\omega_{j}\right) h / 2\right)\right|+\frac{\delta h}{2} \leqslant \widehat{\varepsilon} h+\frac{\delta h}{2} \leqslant \delta h,
\end{aligned}
$$

by Assumption 2 and for $2 \delta \leqslant 1$. The nonresonance condition of Assumption 2 (used with $\mathbf{k}-\langle j\rangle$ in place of $\mathbf{k}$ ) thus implies that

$$
|j|^{s-\widehat{s}} \leqslant c_{2}^{(s-\widehat{s}) / 2} \delta^{N}\left(\Gamma^{\mathbf{k}}\right)^{s-\widehat{s}}
$$

for $s-\widehat{s} \geqslant 2 s_{2}$. This shows that

$$
\|\mathbf{e}\|_{s, \tau} \leqslant c_{2}^{(s-\widehat{s}) / 2} \delta^{N}\left\|\boldsymbol{\Gamma}^{s-\widehat{s}} \mathbf{e}\right\|_{\widehat{s}, \tau},
$$

and the claimed estimate of $\mathbf{e}$ follows from Lemma 7.

Now, we study the difference $\xi^{n}-\xi\left(t_{n}\right)$ of the numerical solution $\xi^{n}$ of (22) and its modulated Fourier expansion $\xi(t)$ of (32). In this modulated Fourier expansion $\xi(t)$ of (32) we use the modulation functions constructed in (38) at discrete times $t_{n}=n h$.

Proposition 9. We have, for $\delta \leqslant \delta_{0}$ and $s \geqslant s_{0}$,

$$
\left\|\xi^{n}-\xi\left(t_{n}\right)\right\|_{s} \leqslant C \varepsilon \delta^{N} \quad \text { for } 0 \leqslant t_{n}=n h \leqslant \delta^{-1}
$$

with constants $C, s_{0}$, and $\delta_{0}$.

Proof. (a) From Lemma 7, we know for the modulated Fourier expansion the estimate

$$
\left\|\xi\left(t_{n}\right)\right\|_{s} \leqslant C \varepsilon \quad \text { for } 0 \leqslant t_{n} \leqslant \delta^{-1}
$$


(b) A corresponding estimate holds, for sufficiently small $\varepsilon$, also for the numerical solution:

$$
\left\|\xi^{n}\right\|_{s} \leqslant 2 \widehat{c}^{-1} \varepsilon \quad \text { for } 0 \leqslant t_{n} \leqslant \varepsilon^{-1 / 2}
$$

with $\widehat{c}$ from (30), since, by Lemma 6 and induction,

$$
\left\|\xi^{n+1}\right\|_{s} \leqslant\left\|\xi^{0}\right\|_{s}+h \sum_{n^{\prime}=0}^{n} \sum_{m+m^{\prime}=2}^{\infty} C_{m, m^{\prime}, s}\left\|\xi^{n^{\prime}}\right\|_{s}^{m+m^{\prime}} \leqslant \widehat{c}^{-1} \varepsilon+\frac{4 C}{\widehat{c}^{2} r^{2}} n h \varepsilon^{2}
$$

for $2 \varepsilon \leqslant r \widehat{c}$ with $C$ and $r$ from Lemma 6. We may assume without loss of generality that the constant $C$ from (a) is larger than $2 / \widehat{c}$.

(c) The modulated Fourier expansion satisfies, by (42),

$$
\begin{aligned}
\xi_{j}\left(t_{n+1}\right)= & e^{-i \omega_{j} h} \xi_{j}\left(t_{n}\right)-\sum_{\mathbf{k}}\left(d_{j}^{\mathbf{k}}\left(\delta t_{n}\right)+e_{j}^{\mathbf{k}}\left(\delta t_{n}\right)\right) e^{-i(\mathbf{k} \cdot \varpi) t} \\
& +\sum_{m+m^{\prime}=2}^{\infty} \sum_{k \in \mathcal{Z}^{m}, l \in \mathcal{Z}^{m^{\prime}}} h Q_{j, k, l} \xi_{k^{1}}\left(t_{n}\right) \cdots \xi_{k^{m}}\left(t_{n}\right) \bar{\xi}_{l^{1}}\left(t_{n}\right) \cdots \bar{\xi}_{l^{m^{\prime}}}\left(t_{n}\right) .
\end{aligned}
$$

Subtracting the numerical solution $\xi^{n+1}$ of (22), and using (a), (b), and Lemmas 6,8 shows that, for $0 \leqslant t_{n} \leqslant \delta^{-1}$, and for sufficiently small $\varepsilon$,

$$
\left\|\xi^{n+1}-\xi\left(t_{n+1}\right)\right\|_{s} \leqslant\left\|\xi^{n}-\xi\left(t_{n}\right)\right\|_{s}+C \varepsilon \delta^{N+1} h+C \varepsilon h\left\|\xi^{n}-\xi\left(t_{n}\right)\right\|_{s} .
$$

The claimed estimate follows inductively.

4.6. The splitting structure of the modulated Fourier expansion. In the previous subsections, a modulated Fourier expansion was constructed and analyzed based on the representation (22) ofthe numerical scheme, that is, based on flows of differential equations. Recall that we have derived in Section 3.5 differential equations (and Hamiltonian functions) that underlie the flows that compose the numerical scheme. In this subsection, we will derive corresponding differential equations for the modulated Fourier expansion.

Motivated by (27), we denote by $\Phi_{\mathbf{H}_{0}}^{h}$ the flow at time $h$ of the Hamiltonian differential equation

$$
i \dot{z}_{j}^{\mathbf{k}}=\frac{\partial \mathbf{H}_{0}}{\partial \bar{z}_{j}^{\mathbf{k}}}(\mathbf{z}, \overline{\mathbf{z}})
$$

with Hamiltonian function

$$
\mathbf{H}_{0}(\mathbf{z}, \overline{\mathbf{z}})=\sum_{\mathbf{k}} \sum_{j \in \mathcal{Z}}\left(|\ell+j \bmod 2 K|^{2}-|\ell|^{2}\right)\left|w_{j}^{\mathbf{k}}\right|^{2}, \quad\left(\begin{array}{c}
w_{j}^{\mathbf{k}} \\
\bar{w}_{-j}^{-\mathbf{k}}
\end{array}\right)=S_{j}^{-1}\left(\begin{array}{c}
z_{j}^{\mathbf{k}} \\
\bar{z}_{-j}^{-\mathbf{k}}
\end{array}\right) .
$$


Correspondingly, we denote by $\Phi_{\mathbf{P}}^{h}$ the flow at time $h$ of the Hamiltonian differential equation with Hamiltonian function

$$
\mathbf{P}(\mathbf{z}, \overline{\mathbf{z}})=\sum_{m+m^{\prime}=0}^{\infty} \sum_{\substack{\mathbf{k}^{1}+\ldots+\mathbf{k}^{m} \\-1^{1}-\cdots-m^{m^{\prime}} \in \mathcal{M}}} \sum_{k \in \mathcal{Z}^{m}, l \in \mathcal{Z}^{m^{\prime}}} P_{k, l} z_{k^{1}}^{\mathbf{k}^{1}} \cdots z_{k^{m}}^{\mathbf{k}^{m}} \bar{z}_{l^{1}}^{1^{1}} \cdots \bar{z}_{l^{m^{\prime}}}^{m^{m^{\prime}}} ;
$$

compare (29).

The splitting structure of the modulation system for the modulation functions $\mathbf{z}$ is revealed in the following lemma: advancing the modulation functions by $\delta h$ corresponds, up to a small defect, to solving Hamiltonian differential equations with Hamiltonian functions $\mathbf{H}_{0}$ and $\mathbf{P}$ one after another.

LEMMA 10. We have

$$
\Phi_{\mathbf{H}_{0}}^{h} \circ \Phi_{\mathbf{P}}^{h}\left(\mathbf{z}\left(\delta t_{n}\right)\right)=\widetilde{\mathbf{z}}\left(\delta t_{n+1}\right)+\mathbf{d}\left(\delta t_{n}\right)+\mathbf{e}\left(\delta t_{n}\right)
$$

with the defects $\mathbf{d}$ and $\mathbf{e}$ of (42)-(44), and where $\widetilde{z}_{j}^{\mathbf{k}}\left(\delta t_{n+1}\right)=z_{j}^{\mathbf{k}}\left(\delta t_{n+1}\right) e^{-i(\mathbf{k} \cdot \boldsymbol{\omega}) h}$.

Proof. Let

$$
\left(\Phi_{P}^{h}(\xi)\right)_{j}=\sum_{m+m^{\prime}=0}^{\infty} \sum_{k \in \mathcal{Z}^{m}, l \in \mathcal{Z}^{m^{\prime}}} P_{j, k, l} \xi_{k^{1}} \cdots \xi_{k^{m}} \bar{\xi}_{l^{1}} \cdots \bar{\xi}_{l^{m^{\prime}}}
$$

be the expansion of the flow $\Phi_{P}^{h}$ given by (28). Then one verifies that the flow $\Phi_{\mathbf{P}}^{h}$ is given by the same coefficients $P_{j, k, l}$,

$$
\left(\Phi_{\mathbf{P}}^{h}(\mathbf{z})\right)_{j}^{\mathbf{k}}=\sum_{m+m^{\prime}=0}^{\infty} \sum_{k \in \mathcal{Z}^{m}, l \in \mathcal{Z}^{m^{\prime}}} \sum_{\substack{\left.\mathbf{k}^{1}+\ldots+\mathbf{k}^{m} \\-\mathbf{1}^{1}-\ldots-\mathbf{l}^{m^{\prime}} \in \mathbf{k}\right]}} P_{j, k, l} z_{k^{1}}^{\mathbf{k}^{1}} \cdots z_{k^{m}}^{\mathbf{k}^{m}} \bar{z}_{l^{1}}^{1^{1}} \cdots \bar{z}_{l^{m^{\prime}}}^{\mathbf{l}^{m^{\prime}}} .
$$

This implies that also the coefficients of the expansions of $\Phi_{H_{0}}^{h} \circ \Phi_{P}^{h}(\xi)$ and $\Phi_{\mathbf{H}_{0}}^{h} \circ$ $\Phi_{\mathbf{P}}^{h}(\mathbf{z})$ coincide. The coefficients in the expansion of $\Phi_{H_{0}}^{h} \circ \Phi_{P}^{h}(\xi)$ are given by (22), and they also appear in the expansion (42) of $\widetilde{\mathbf{z}}\left(\delta t_{n+1}\right)+\mathbf{d}\left(\delta t_{n}\right)+\mathbf{e}\left(\delta t_{n}\right)$. The statement of the lemma follows.

4.7. Discrete almost-invariants. An essential property of modulated Fourier expansions is the existence of formal invariants. These invariants will finally allow us to consider long time intervals by patching together many of the short time intervals considered so far. They take the form

$$
\mathcal{I}_{m}(\mathbf{z})=\sum_{\mathbf{k}} \sum_{j \in \mathcal{Z}} \sum_{\substack{l \in \mathcal{Z} \\ n(l)=m}} k_{l}\left|z_{j}^{\mathbf{k}}\right|^{2} \quad \text { for } m \in \mathcal{N}:=\{n(j): j \in \mathcal{Z}\} .
$$


This is well defined (recall that the $\sum_{\mathbf{k}}$ stands for the sum over the equivalence classes $\left.[\mathbf{k}] \in \mathbb{Z}^{\mathcal{Z}} / \mathcal{M}\right)$, since $\sum_{l: n(l)=m} k_{l}=0$ for $\mathbf{k} \in \mathcal{M}$ by part (iii) of Assumption 2.

LEMMA 11. We have

$$
\mathcal{I}_{m}\left(\Phi_{\mathbf{H}_{0}}^{h} \circ \Phi_{\mathbf{P}}^{h}\left(\mathbf{z}\left(\delta t_{n}\right)\right)\right)=\mathcal{I}_{m}\left(\mathbf{z}\left(\delta t_{n}\right)\right) \quad \text { for } m \in \mathcal{N} .
$$

Proof. Let $\mathbf{S}(\theta)$ be defined by

$$
(\mathbf{S}(\theta) \mathbf{z})_{j}^{\mathbf{k}}=e^{i \theta \sum_{l: n(l)=m} k_{l}} z_{j}^{\mathbf{k}}
$$

for $m \in \mathcal{N}$. The Hamiltonian function $\mathbf{P}$ from the previous subsection is invariant under the transformations $\mathbf{S}(\theta)$, and this leads to conserved quantities by Noether's theorem. Along a solution $\mathbf{z}=\Phi_{\mathbf{P}}^{t}\left(\mathbf{z}^{0}\right)$ of the Hamiltonian differential equation with Hamiltonian function $\mathbf{P}$, we have

$$
\begin{aligned}
0 & =\left.\frac{d}{d \theta}\right|_{\theta=0} \mathbf{P}(\mathbf{S}(\theta) \mathbf{z}, \overline{\mathbf{S}(\theta) \mathbf{z}}) \\
& =-i \sum_{\mathbf{k}} \sum_{j \in \mathcal{Z}} \sum_{\substack{l \in \mathcal{Z} \\
n(l)=m}} k_{l}\left(\bar{z}_{j}^{\mathbf{k}} \frac{\partial \mathbf{P}}{\partial \bar{z}_{j}^{\mathbf{k}}}(\mathbf{z}, \overline{\mathbf{z}})-z_{j}^{\mathbf{k}} \frac{\partial \mathbf{P}}{\partial z_{j}^{\mathbf{k}}}(\mathbf{z}, \overline{\mathbf{z}})\right) \\
& =\sum_{\mathbf{k}} \sum_{j \in \mathcal{Z}} \sum_{\substack{l \in \mathcal{Z} \\
n(l)=m}} k_{l} \frac{d}{d t}\left|z_{j}^{\mathbf{k}}\right|^{2}=\frac{d}{d t} \mathcal{I}_{m}(\mathbf{z}) .
\end{aligned}
$$

This implies conservation of $\mathcal{I}_{m}$ along the flow of $\mathbf{P}$,

$$
\mathcal{I}_{m}\left(\Phi_{\mathbf{P}}^{h}\left(\mathbf{z}\left(\delta t_{n}\right)\right)\right)=\mathcal{I}_{m}\left(\mathbf{z}\left(\delta t_{n}\right)\right)
$$

In the same way, one shows conservation of $\mathcal{I}_{m}$ along the flow of $\mathbf{H}_{0}$, and the statement of the lemma follows.

In the end, we are interested more in $\mathbf{z}\left(\delta t_{n+1}\right)$ than in $\Phi_{\mathbf{H}_{0}}^{h} \circ \Phi_{\mathbf{P}}^{h}\left(\mathbf{z}\left(\delta t_{n}\right)\right)$. The following proposition shows that $\mathcal{I}_{m}$ is an almost-invariant along the modulation functions $\mathbf{z}$.

Proposition 12. We have, for $\delta \leqslant \delta_{0}$ and $s \geqslant s_{0}$,

$$
\sum_{m \in \mathcal{N}} \max (1, m)^{s}\left|\mathcal{I}_{m}\left(\mathbf{z}\left(\delta t_{n}\right)\right)-\mathcal{I}_{m}(\mathbf{z}(0))\right| \leqslant C \varepsilon^{2} \delta^{N} \quad \text { for } 0 \leqslant t_{n}=n h \leqslant \delta^{-1}
$$

with constants $C, s_{0}$, and $\delta_{0}$. 
Proof. Throughout the proof, we work with the representative $\mathbf{k}$ of [k] for which the minimum in the definition (41) of $\Gamma^{\mathbf{k}}$ is attained.

(a) By Lemmas 10 and 11, we have

$$
\left|\mathcal{I}_{m}\left(\mathbf{z}\left(\delta t_{n+1}\right)\right)-\mathcal{I}_{m}\left(\mathbf{z}\left(\delta t_{n}\right)\right)\right| \leqslant 2 \sum_{\mathbf{k}} \sum_{j \in \mathcal{Z}} \sum_{\substack{l \in \mathcal{Z} \\ n(l)=m}}\left|k_{l}\right|\left(\left|z_{j}^{\mathbf{k}}\right|\left|d_{j}^{\mathbf{k}}\right|+\left|d_{j}^{\mathbf{k}}\right|^{2}+\left|e_{j}^{\mathbf{k}}\right|^{2}\right),
$$

since $z_{j}^{\mathbf{k}}=0$ if $e_{j}^{\mathbf{k}} \neq 0$. Here, the modulation functions $z_{j}^{\mathbf{k}}$ on the right-hand side are evaluated at time $\tau=\delta t_{n+1}$ and the defects $d_{j}^{\mathbf{k}}$ and $e_{j}^{\mathbf{k}}$ at time $\tau=\delta t_{n}$.

(b) Let $\mathbf{k} \neq \mathbf{0}$ and $j=j(\mathbf{k})=\sum_{l \in \mathcal{Z}} k_{l} l \bmod 2 M \in \mathcal{Z}$, and let $\bar{l} \in \mathcal{Z}$ be the index of largest norm $|\cdot|$ with $k_{\bar{l}} \neq 0$. Then we have

$$
|\bar{l}|^{2} \leqslant|j| \cdot \Gamma^{\mathbf{k}} .
$$

Indeed, if $|\bar{l}|^{2}>\Gamma^{\mathbf{k}}$, then necessarily $\left|k_{\bar{l}}\right|=1$ and $\|\mathbf{k}\| \cdot|l| \leqslant|\bar{l}|$ for all $l \neq \bar{l}$ with $k_{l} \neq 0$, and hence

$$
|\bar{l}| \leqslant\left|j-\sum_{\bar{l} \neq l \in \mathcal{Z}} k_{l} l\right| \leqslant|j|+\frac{\|\mathbf{k}\|-1}{\|\mathbf{k}\|}|\bar{l}|
$$

that is, $|\bar{l}| \leqslant\|\mathbf{k}\| \cdot|j|$. This implies for $s \geqslant 2 \widehat{s}$ that

$$
\sum_{l \in \mathcal{Z}}\left|k_{l}\right||l|^{2 s} \leqslant\|\mathbf{k}\| \cdot|\bar{l}|^{2 s} \leqslant|j|^{2 \widehat{s}}\left(\Gamma^{\mathbf{k}}\right)^{2(s-\widehat{s})} .
$$

The last estimate is improved for near-resonant indices (for which $e_{j}^{\mathbf{k}} \neq 0$ ) by the nonresonance condition in Assumption 2 to

$$
\sum_{l \in \mathcal{Z}}\left|k_{l}\right||l|^{2 s} \leqslant c_{2}^{s-2 \widehat{s}}|j|^{2 \widehat{s}}\left(\Gamma^{\mathbf{k}}\right)^{2(s-\widehat{s})} \delta^{N}
$$

if $s-2 \widehat{s} \geqslant s_{2}$.

(c) By (a), (b), the Cauchy-Schwarz inequality, and Lemma 13 below, we have

$$
\begin{aligned}
& \sum_{m \in \mathcal{N}} \max (1, m)^{s}\left|\mathcal{I}_{m}\left(\mathbf{z}\left(\delta t_{n+1}\right)\right)-\mathcal{I}_{m}\left(\mathbf{z}\left(\delta t_{n}\right)\right)\right| \\
& \quad \leqslant C\left\|\boldsymbol{\Gamma}^{s-\widehat{s}} \mathbf{z}\right\|_{\widehat{s}}\left\|\boldsymbol{\Gamma}^{s-\widehat{s}} \mathbf{d}\right\|_{\widehat{s}}+C\left\|\boldsymbol{\Gamma}^{s-\widehat{s}} \mathbf{d}\right\|_{\widehat{s}}^{2}+C c_{2}^{s-2 \widehat{s}} \delta^{N}\left\|\boldsymbol{\Gamma}^{s-\widehat{s}} \mathbf{e}\right\|_{\widehat{s}}^{2} .
\end{aligned}
$$

The statement of the proposition thus follows from Lemmas 7 and 8 by summing up. 
LEMMA 13. We have

$$
\frac{1}{C}|j|^{2} \leqslant \max (1,|n(j)|) \leqslant C|j|^{2} \quad \text { for all } j \in \mathcal{Z}
$$

with a positive constant $C$ depending only on $\ell$.

Proof. We have $-|\ell|^{2} \leqslant n(j) \leqslant|j|^{2}$, since $|\ell \pm j \bmod 2 K| \leqslant|\ell \pm j|$, and hence $|n(j)| \leqslant C|j|^{2}$. To get a lower bound for $|n(j)|$, we note that $\left|\ell_{1} \pm j_{1} \bmod 2 K\right| \geqslant$ $\min \left(\left|\ell_{1}+j_{1}\right|,\left|\ell_{1}-j_{1}\right|\right)$, and hence

$$
\frac{1}{2}\left|\ell_{1}+j_{1} \bmod 2 K\right|^{2}+\frac{1}{2}\left|\ell_{1}-j_{1} \bmod 2 K\right|^{2}-\ell_{1}^{2} \geqslant j_{1}^{2}-2\left|j_{1}\right| \cdot\left|\ell_{1}\right| .
$$

This holds not only for the first component, and by summing up all components we get

$$
n(j) \geqslant|j|^{2}-2|\ell| \cdot|j| \text {. }
$$

We therefore get $n(j) \geqslant \frac{1}{2}|j|^{2}$ for $4|\ell|<|j|$. For $4|\ell| \geqslant|j|$, we have $\max (1,|n(j)|) \geqslant 1 \geqslant \frac{1}{C}|j|^{2}$.

Next, we show that the almost-invariants $\mathcal{I}_{m}$ of (45) are close to the superactions

$$
I_{m}(\xi)=\sum_{\substack{l \in \mathcal{Z} \\ n(l)=m}}\left|\xi_{l}\right|^{2}, \quad m \in \mathcal{N}
$$

that collect those actions $\left|\xi_{l}\right|^{2}$ with the same value $n(l)$.

Proposition 14. We have, for $\delta \leqslant \delta_{0}$ and $s \geqslant s_{0}$,

$$
\sum_{m \in \mathcal{N}} \max (1, m)^{s}\left|\mathcal{I}_{m}\left(\mathbf{z}\left(\delta t_{n}\right)\right)-I_{m}\left(\xi^{n}\right)\right| \leqslant C \varepsilon^{2} \delta \quad \text { for } 0 \leqslant t_{n}=n h \leqslant \delta^{-1}
$$

with constants $C, s_{0}$, and $\delta_{0}$.

Proof. We omit the argument $\delta t_{n}$ of the modulation functions. We have, by (38a),

$$
\mathcal{I}_{m}(\mathbf{z})-\sum_{\substack{l \in \mathcal{Z}: \\ n(l)=m}}\left|z_{l}^{\langle l\rangle}\right|^{2}=\sum_{\substack{l \in \mathcal{Z}: \\ n(l)=m}} \sum_{\mathbf{k} \neq\langle l\rangle} k_{l}\left|z_{j(\mathbf{k})}^{\mathbf{k}}\right|^{2},
$$

and part (b) of the proof of Proposition 12 together with Lemma 7, Lemma 13, and (39) implies that

$$
\left.\sum_{m \in \mathcal{N}} \max (1, m)^{s}\left|\mathcal{I}_{m}(\mathbf{z})-\sum_{\substack{l \in \mathcal{Z}: \\ n(l)=m}}\right| z_{l}^{\langle l\rangle}\right|^{2} \mid \leqslant C \varepsilon^{2} \delta^{2} .
$$


On the other hand, we have, for the modulated Fourier expansions $\xi(t)$ (32),

$$
\left.\left|I_{m}\left(\xi\left(t_{n}\right)\right)-\sum_{\substack{l \in \mathcal{Z}: \\ n(l)=m}}\right| z_{l}^{\langle l\rangle}\right|^{2} \mid \leqslant 2 \sum_{\substack{l \in \mathcal{Z}: \\ n(l)=m}}\left(\sum_{\mathbf{k} \neq\langle l\rangle}\left|z_{l}^{\mathbf{k}}\right|\right)\left(\sum_{\mathbf{k}}\left|z_{l}^{\mathbf{k}}\right|\right),
$$

and hence, by the Cauchy-Schwarz inequality together with Lemma 7, Lemma 13, and (39),

$$
\left.\sum_{m \in \mathcal{N}} \max (1, m)^{s}\left|I_{m}\left(\xi\left(t_{n}\right)\right)-\sum_{\substack{l \in \mathcal{Z} \\ n(l)=m}}\right| z_{l}^{\langle l\rangle}\right|^{2} \mid \leqslant C \varepsilon^{2} \delta .
$$

Finally, we have, by Proposition 9 and Lemma 13, for the numerical solution $\xi^{n}$,

$$
\sum_{m \in \mathcal{N}} \max (1, m)^{s}\left|I_{m}\left(\xi\left(t_{n}\right)\right)-I_{m}\left(\xi^{n}\right)\right| \leqslant C \varepsilon^{2} \delta^{N}
$$

where we have used that $\left.|| \xi_{j}\left(t_{n}\right)\right|^{2}-\left|\xi_{j}^{n}\right|^{2}|\leqslant| \xi_{j}\left(t_{n}\right)-\xi_{j}^{n} \mid\left(\left|\xi_{j}\left(t_{n}\right)\right|+\left|\xi_{j}^{n}\right|\right)$. Putting all this together proves the statement of the proposition.

4.8. Modulated Fourier expansion on another time interval. All estimates of the previous subsections are valid on the time interval $0 \leqslant t_{n}=n h \leqslant \delta^{-1}$. We assume without loss of generality that

$$
\delta^{-1}=\tilde{n} h
$$

for some $\tilde{n} \in \mathbb{N}$. In this subsection, we consider consecutive short time intervals

$$
\nu \delta^{-1} \leqslant t_{n}=n h \leqslant(\nu+1) \delta^{-1} \text { for } v=0,1,2, \ldots
$$

In principle, we can repeat the construction of a modulated Fourier expansion described in Sections 4.2-4.3 on these time intervals, taking $\xi^{\nu \widetilde{n}}$ as initial value instead of $\xi^{0}$. This gives us modulation functions $\mathbf{z}^{v}$ on the $v$ th time interval constructed in such a way that

$$
\xi_{j}^{n} \approx \xi_{j}^{v}\left(t_{n}\right)=\sum_{\mathbf{k}} z_{j}^{\mathbf{k}, v}\left(\delta t_{n}\right) e^{-i(\mathbf{k} \cdot \boldsymbol{\varpi}) t_{n}} \quad \text { for } \nu \delta^{-1} \leqslant t_{n} \leqslant(\nu+1) \delta^{-1} .
$$

The estimates of Sections 4.4-4.7 remain valid provided that $\xi^{\nu \widetilde{n}}$ satisfies the smallness condition (up to a constant) as $\xi^{0}$ in (30). In the following lemma, we bound the difference of the modulated Fourier expansions $\mathbf{z}^{v}$ and $\mathbf{z}^{v-1}$ at the interface $\delta t_{v \widetilde{n}}$ of their intervals of validity. 
LEMma 15. Assume that, for $v \geqslant 1$,

$$
\left\|\xi^{(\nu-1) \tilde{n}}\right\|_{s} \leqslant 2 \widehat{c}^{-1} \varepsilon \quad \text { and } \quad\left\|\xi^{\nu \tilde{n}}\right\|_{s} \leqslant 2 \widehat{c}^{-1} \varepsilon .
$$

Then we have, for $\delta \leqslant \delta_{0}$ and $s \geqslant s_{0}$,

$$
\left\|\boldsymbol{\Gamma}^{s-\widehat{s}} \mathbf{z}^{\nu}\left(\delta t_{v \widetilde{n}}\right)-\boldsymbol{\Gamma}^{s-\widehat{s}} \mathbf{z}^{\nu-1}\left(\delta t_{v \widetilde{n}}\right)\right\|_{\widehat{s}} \leqslant C \varepsilon \delta^{N}
$$

with constants $C, s_{0}$, and $\delta_{0}$.

Proof. Let $n=v \widetilde{n}$.

(a) We first show by induction on $q=0, \ldots, N$ that

$$
M_{q}^{v}(\mathbf{z}):=\left\|\sum_{p=0}^{q} \varepsilon \delta^{p}\left(\mathbf{z}_{p}^{v}\left(\delta t_{n}\right)-\mathbf{z}_{p}^{v-1}\left(\delta t_{n}\right)\right)\right\| \|_{s} \leqslant C \varepsilon \delta^{q},
$$

where $\mathbf{z}_{p}^{v}=\left(z_{j, p}^{\mathbf{k}, v}\right)_{j \in \mathcal{Z}, \mathbf{k} \in \mathbb{Z}^{z}}$. For this purpose, we split the modulation functions, $\mathbf{z}_{p}=\mathbf{a}_{p}+\mathbf{b}_{p}$ with $a_{j, p}^{\langle j\rangle}=z_{j, p}^{\langle j\rangle}$ and $b_{j, p}^{\mathbf{k}}=z_{j, p}^{\mathbf{k}}$ for $\mathbf{k} \neq\langle j\rangle$.

We consider the nonlinearities $F_{j, p}^{\mathbf{k}, v}$ and $F_{j, p}^{\mathbf{k}, v-1}$ on the right-hand sides of (36a). By Lemmas 6 and 7, we have

$$
M_{q}^{v}(\mathbf{F}) \leqslant C \varepsilon \delta^{-1} M_{q-1}^{v}(\mathbf{z}),
$$

and hence, by construction (38b) and (38e),

$$
M_{q}^{v}(\mathbf{b}) \leqslant C \delta M_{q-1}^{v}(\mathbf{z}) \quad \text { and } \quad M_{q}^{v}(\dot{\mathbf{a}}) \leqslant C \delta M_{q-1}^{v}(\mathbf{z}) .
$$

In order to complete the inductive proof of (48), we need a similar estimate also for $M_{q}^{v}(\mathbf{a})$. Note that, by construction (38d) of $\mathbf{z}^{v}$,

$$
\sum_{\mathbf{k}} \sum_{p=0}^{q} \varepsilon \delta^{p} z_{j, p}^{\mathbf{k}, v}\left(\delta t_{n}\right) e^{-i(\mathbf{k} \cdot \boldsymbol{\varpi}) t_{n}}=\sum_{\mathbf{k}} \sum_{p=0}^{q} \varepsilon \delta^{p} z_{j, p}^{\mathbf{k}, v-1}\left(\delta t_{n}\right) e^{-i(\mathbf{k} \cdot \varpi) t_{n}}+r_{j}^{n}
$$

with $\left\|r^{n}\right\|_{s} \leqslant C \varepsilon \delta^{q}$ by Proposition 9 (applied on the $(v-1)$ th interval with modulation functions $z_{j}^{\mathbf{k}, v-1}$ truncated after $p=q$ instead of $p=N$ as in (38f)). This shows that

$$
\left(\sum_{j \in \mathcal{Z}}|j|^{2 s}\left|\sum_{p=0}^{q} \varepsilon \delta^{p}\left(a_{j, p}^{\langle j\rangle, v}\left(\delta t_{n}\right)-a_{j, p}^{\langle j\rangle, v-1}\left(\delta t_{n}\right)\right)\right|^{2}\right)^{1 / 2} \leqslant M_{q}^{v}(\mathbf{b})+C \varepsilon \delta^{q},
$$

and hence, by (49),

$$
M_{q}^{v}(\mathbf{a}) \leqslant C \delta M_{q-1}^{v}(\mathbf{z})+C \varepsilon \delta^{q} .
$$


This completes the proof of (48).

(b) In order to prove the statement of the lemma, we have to consider

$$
\widehat{M}_{q}^{v}(\mathbf{z}):=\left\|\sum_{p=0}^{q} \varepsilon \delta^{p} \boldsymbol{\Gamma}^{s-\widehat{s}}\left(\mathbf{z}_{p}^{v}\left(\delta t_{n}\right)-\mathbf{z}_{p}^{v-1}\left(\delta t_{n}\right)\right)\right\| \|_{\widehat{s}}
$$

instead of $M_{q}^{v}(\mathbf{z})$. Note that $\widehat{M}_{q}^{v}(\mathbf{a})=M_{q}^{v}(\mathbf{a})$, and that the estimates (49) transfer to $\widehat{M}_{q}^{v}$ by (41). This finishes the proof of the lemma.

The following proposition bounds, in the situation of the above lemma, the difference of the almost-invariants $\mathcal{I}_{m}$ (45) at the interface of two time intervals.

Proposition 16. Assume that, for $v \geqslant 1$,

$$
\left\|\xi^{(\nu-1) \widetilde{n}}\right\|_{s} \leqslant 2 \widehat{c}^{-1} \varepsilon \quad \text { and } \quad\left\|\xi^{\nu \widetilde{n}}\right\|_{s} \leqslant 2 \widehat{c}^{-1} \varepsilon .
$$

Then we have, for $\delta \leqslant \delta_{0}$ and $s \geqslant s_{0}$,

$$
\sum_{m \in \mathcal{N}} \max (1, m)^{s}\left|\mathcal{I}_{m}\left(\mathbf{z}^{v}\left(\delta t_{v \tilde{n}}\right)\right)-\mathcal{I}_{m}\left(\mathbf{z}^{\nu-1}\left(\delta t_{v \tilde{n}}\right)\right)\right| \leqslant C \varepsilon^{2} \delta^{N}
$$

with constants $C, s_{0}$, and $\delta_{0}$.

Proof. This follows using part (b) of the proof of Proposition 12, the CauchySchwarz inequality, and the estimates of Lemmas 7, 13, and 15.

4.9. Long-time near-conservation of super-actions. We put the results of all previous subsections together to show near-conservation of the super-actions (46) on long time intervals of length $\delta^{-N}$.

THEOREM 17. The super-actions are nearly conserved for $\delta \leqslant \delta_{0}$ and $s \geqslant s_{0}$ :

$$
\sum_{m \in \mathcal{N}} \max (1, m)^{s}\left|I_{m}\left(\xi^{n}\right)-I_{m}\left(\xi^{0}\right)\right| \leqslant C \varepsilon^{2} \delta \quad \text { for } 0 \leqslant t_{n}=n h \leqslant \delta^{-N}
$$

with constants $C, s_{0}$ and $\delta_{0}$.

Proof. Let $C / 5$ be the maximum of the constant of Proposition 16 and the constants that appear in Propositions 12 and 14 if $\widehat{c}^{-1}$ in (30) is replaced by $2 \widehat{c}^{-1}$.

With this constant $C$, we prove the theorem for $v \delta^{-1} \leqslant t_{n} \leqslant(\nu+1) \delta^{-1}$ and $v=0,1,2, \ldots$ by induction on $v$. The main observation is that, for $v \delta^{-1} \leqslant t_{n} \leqslant$ $(v+1) \delta^{-1}$, and with $\tilde{n}=1 /(\delta h)$ as in (47),

$$
\left|I_{m}\left(\xi^{n}\right)-I_{m}\left(\xi^{0}\right)\right| \leqslant\left|I_{m}\left(\xi^{n}\right)-\mathcal{I}_{m}\left(\mathbf{z}^{v}\left(\delta t_{n}\right)\right)\right|+\left|\mathcal{I}_{m}\left(\mathbf{z}^{v}\left(\delta t_{n}\right)\right)-\mathcal{I}_{m}\left(\mathbf{z}^{v}\left(\delta t_{v \tilde{n}}\right)\right)\right|
$$




$$
\begin{aligned}
& +\sum_{\widetilde{v}=1}^{v}\left|\mathcal{I}_{m}\left(\mathbf{z}^{\widetilde{v}}\left(\delta t_{\widetilde{v} \tilde{n}}\right)\right)-\mathcal{I}_{m}\left(\mathbf{z}^{\widetilde{v}-1}\left(\delta t_{\widetilde{v} \tilde{n}}\right)\right)\right| \\
& +\sum_{\widetilde{v}=1}^{v}\left|\mathcal{I}_{m}\left(\mathbf{z}^{\widetilde{v}-1}\left(\delta t_{\widetilde{v} \tilde{n}}\right)\right)-\mathcal{I}_{m}\left(\mathbf{z}^{\widetilde{v}-1}\left(\delta t_{(\widetilde{v}-1) \tilde{n}}\right)\right)\right| \\
& +\left|\mathcal{I}_{m}\left(\mathbf{z}^{0}(0)\right)-I_{m}\left(\xi^{0}\right)\right|
\end{aligned}
$$

After multiplying by $\max (1, m)^{s}$ and summing over $m \in \mathcal{N}$, we can apply Propositions 12, 14, and 16 to the different terms in (51), since, when $v \geqslant 1$, the induction hypothesis implies that, for $0 \leqslant n \leqslant v \widetilde{n}$,

$$
\begin{aligned}
\left\|\xi^{n}\right\|_{s}^{2} & \leqslant\left\|\xi^{0}\right\|_{s}^{2}+C_{1}^{s} \sum_{m \in \mathcal{N}} \max (1, m)^{s}\left|I_{m}\left(\xi^{n}\right)-I_{m}\left(\xi^{0}\right)\right| \\
& \leqslant \widehat{c}^{-2} \varepsilon^{2}+C_{1}^{s} C \varepsilon^{2} \delta \leqslant 2 \widehat{c}^{-2} \varepsilon^{2}
\end{aligned}
$$

provided that $\delta \leqslant 1 /\left(C_{1}^{s} C \widehat{c}^{2}\right)$ with the constant $C_{1}$ of Lemma 13 . This gives

$$
\sum_{m \in \mathcal{N}} \max (1, m)^{s}\left|I_{m}\left(\xi^{n}\right)-I_{m}\left(\xi^{0}\right)\right| \leqslant \frac{2}{5} C \varepsilon^{2} \delta+\frac{1}{5} C(2 v+1) \varepsilon^{2} \delta^{N}
$$

The statement of the theorem follows for $v \leqslant \delta^{-N+1}$, that is, for $t_{n} \leqslant \delta^{-N}$.

4.10. Proof of Theorem 1. In order to complete the proof of Theorem 1 , we go in a final step back from the new variables $\xi$ introduced in Section 3 to the original variables $u$, in which the split-step Fourier method (4) is formulated. Under the conditions, and with the constant $C$ of Theorem 17 , we have, for $0 \leqslant t_{n}=n h \leqslant$ $\delta^{-N}$ and $\delta \leqslant 1 /\left(C_{1}^{s}, C \widehat{c}^{2}\right)$

$$
\left\|\xi^{n}\right\|_{s}^{2} \leqslant\left\|\xi^{0}\right\|_{s}^{2}+C_{1}^{s} \sum_{m \in \mathcal{N}} \max (1, m)^{s}\left|I_{m}\left(\xi^{n}\right)-I_{m}\left(\xi^{0}\right)\right| \leqslant 2 \widehat{c}^{-2} \varepsilon^{2}
$$

with the constant $C_{1}$ of Lemma 13 . By Lemma 5 , this transfers to a statement in the original variables,

$$
\left\|\mathcal{F}_{\neg \ell}\left(u^{m}\right)\right\|_{s} \leqslant \widehat{C}\left\|\xi^{n}\right\|_{s} \leqslant \sqrt{2} \widehat{C} \widehat{c}^{-1} \varepsilon \quad \text { for } 0 \leqslant t_{n}=n h \leqslant \delta^{-N},
$$

as claimed in Theorem 1.

\section{On the nonresonance condition}

In this section, we give the proof of Theorem 3 on a sufficient condition under which Assumptions 1 and 2 in Theorem 1 hold. The first subsection deals with 
the (numerical) linear stability of Assumption 1, while the remaining main part of this section is devoted to the nonresonance condition of Assumption 2.

From now on, we let $\ell=0$. In this case, we have $n(j)=|j|^{2}$, and the frequencies (10) become

$$
\omega_{j}=\frac{\arccos \left(\cos \left(|j|^{2} h\right)-h \lambda \rho^{2} \sin \left(|j|^{2} h\right)\right)}{h \operatorname{sgn}\left(\sin \left(|j|^{2} h\right)+h \lambda \rho^{2} \cos \left(|j|^{2} h\right)\right)}
$$

for $j \in \mathcal{Z}$. We introduce the set of possible values of $n(j)$ :

$$
\mathcal{N}=\{n(j): j \in \mathcal{Z}\}=\left\{|j|^{2}: j \in \mathcal{Z}\right\} .
$$

5.1. Linear stability. We show that Assumption 1 is fulfilled, for $\ell=0$, under the conditions (11) and (12) of Theorem 3.

LEMMA 18. Under the step-size restriction (12), condition (11) of analytical linear stability implies condition (8) of numerical linear stability for $0 \leqslant \rho \leqslant \rho_{0}$ with $c_{1}=c_{1}\left(\rho_{0}\right)$. Moreover,

$$
1-h^{2} \rho^{4}+\frac{2 \lambda \rho^{2}}{\mu_{n}} \geqslant \min \left(\frac{1}{2}, 1+2 \lambda \rho_{0}^{2}\right)>0 \quad \text { for all } n \in \mathcal{N}
$$

with

$$
\mu_{n}=\frac{\sin (n h)}{h \cos (n h)}=\frac{\tan (n h)}{h} .
$$

Proof. We note that, for all $n \in \mathcal{N}$,

$$
0 \leqslant \frac{1}{\tan (n h)} \leqslant \frac{1}{\tan (h)} \leqslant \frac{1}{h}-\frac{h}{3}
$$

by the step-size restriction (12). This yields, for $\lambda=+1$,

$$
1-h^{2} \rho^{4}+\frac{2 \lambda \rho^{2}}{\mu_{n}} \geqslant 1-h^{2} \rho^{4},
$$

and, for $\lambda=-1$,

$$
1-h^{2} \rho^{4}+\frac{2 \lambda \rho^{2}}{\mu_{n}} \geqslant 1-h^{2} \rho^{4}-2 h \rho^{2}\left(\frac{1}{h}-\frac{h}{3}\right) \geqslant\left(1-2 \rho^{2}\right)\left(1+\frac{h^{2} \rho^{2}}{2}\right) .
$$

We hence get (53) for $0 \leqslant \rho \leqslant \rho_{0}$ from (11) and (12).

The estimate (53) together with (12) implies that there exists $c_{1}=c_{1}\left(\rho_{0}\right)$ such that

$$
c_{1} h^{2} \leqslant \sin (n h)^{2}\left(1-h^{2} \rho^{4}+\frac{2 \lambda \rho^{2}}{\mu_{n}}\right)=1-\left(\cos (n h)-h \lambda \rho^{2} \sin (n h)\right)^{2}
$$

for all $n \in \mathcal{N}$, and hence (8) holds. 
5.2. Modified frequencies. Now, we turn to Assumption 2, again for $\ell=0$. We begin by constructing the modified frequencies. The reason that we use modified frequencies in the theory developed in the present paper is that it seems to be very hard to verify the nonresonance condition in part (ii) of Assumption 2 directly for the frequencies $\omega_{j}$ of (52). For the frequencies that show up after the linearization of the nonlinear Schrödinger equation itself around a plane wave, however, a suitable nonresonance condition can be established; see [8, Lemma 2.2]. These frequencies are $\sqrt{|j|^{4}+2 \lambda \rho^{2}|j|^{2}}$, and we therefore seek modified frequencies of a similar form.

We fix $\rho_{0}>0$ with (11) and $h$ and $K$ with (12) for some $N \geqslant 2$, as in Theorem 3. The frequencies $\omega_{j}$ of (52) are considered henceforth as functions of $\sigma=\rho^{2}$ with $0 \leqslant \sigma \leqslant \sigma_{0}:=\rho_{0}^{2}$ :

$$
\omega_{j}=\omega_{j}(\sigma)=\frac{\arccos \left(\cos \left(|j|^{2} h\right)-h \lambda \sigma \sin \left(|j|^{2} h\right)\right)}{h} .
$$

(Note that the step-size restriction (12) together with (53) ensures that the sign of $\omega_{j}$ in (52) is positive.)

The derivative of the frequencies $\omega_{j}$ with respect to $\sigma$ is given by

$$
\frac{d \omega_{j}(\sigma)}{d \sigma}=\frac{\lambda}{\sqrt{1-h^{2} \sigma^{2}+\frac{2 \lambda \sigma}{\mu_{|j|^{2}}}}}
$$

with $\mu_{|j|^{2}}$ from (54), which is positive for $j \in \mathcal{Z}$, by (12). This motivates the definition

$$
\varpi_{j}=\varpi_{j}(\sigma)=|j|^{2}-\mu_{|j|^{2}}+\sqrt{\mu_{|j|^{2}}^{2}+2 \lambda \sigma \mu_{|j|^{2}}}, \quad j \in \mathcal{Z},
$$

of the modified frequencies, since we then have

$$
\frac{d \varpi_{j}(\sigma)}{d \sigma}=\frac{\lambda}{\sqrt{1+\frac{2 \lambda \sigma}{\mu_{|j|^{2}}}}} \text { and } \varpi_{j}(0)=\omega_{j}(0) .
$$

This implies that

$$
\frac{d\left(\omega_{j}-\varpi_{j}\right)(\sigma)}{d \sigma}=\frac{\lambda h^{2} \sigma^{2}}{2\left(1-\xi+\frac{2 \lambda \sigma}{\mu_{|j|^{2}}}\right)^{3 / 2}}
$$

for all $j \in \mathcal{Z}$ and some $0 \leqslant \xi=\xi_{j} \leqslant h^{2} \sigma^{2}$, and hence

$$
\left|\omega_{j}-\varpi_{j}\right| \leqslant C_{2} h^{2} \quad \text { for all } j \in \mathcal{K}
$$

with $C_{2}=C_{2}\left(\sigma_{0}\right)$, by (53). The modified frequencies $\varpi_{j}$ are hence close to the original frequencies $\omega_{j}$, as required in part (i) of Assumption 2. 
5.3. Bambusi's nonresonance condition for the modified frequencies. We study resonances among the modified frequencies $\varpi_{j}$ of (56) derived in the previous subsection. As $\varpi_{j}=\varpi_{l}$ for $|j|^{2}=|l|^{2}$, we introduce

$$
\Omega_{n}=\Omega_{n}(\sigma)=\varpi_{j}(\sigma) \text { for } n \in \mathcal{N}, j \in \mathcal{Z} \text { with } n=|j|^{2} .
$$

We verify for these modified frequencies a nonresonance condition that has been introduced by Bambusi and is widely used in the long-time analysis of infinitedimensional Hamiltonian systems. The verification is an adaptation of [3, Section 5.1] to the present situation, along with some simplifications.

We proceed roughly as follows. The aim is to show that there are a lot of 'good' values of $\sigma$ for which linear combinations of frequencies do not become small. More precisely, a value of $\sigma$ is considered as 'good' if, for all vectors $\mathbf{k} \in \mathbb{Z}^{\mathcal{N}}$ and $\mathbf{l} \in \mathbb{Z}^{\mathcal{N}}$ with $\|\mathbf{k}\|=\sum_{n \in \mathcal{N}}\left|k_{n}\right| \leqslant N$ and $\|\mathbf{I}\| \leqslant 2$, the linear combinations

$$
\mathbf{k} \cdot \boldsymbol{\Omega}+\mathbf{l} \cdot \boldsymbol{\Omega}=\sum_{n \in \mathcal{N}} k_{n} \Omega_{n}+\sum_{n \in \mathcal{N}} l_{n} \Omega_{n}
$$

are bounded away from zero by a negative power of $\operatorname{argmax}(\mathbf{k})$, where we denote by $\operatorname{argmax}(\mathbf{k})$ the largest index $n \in \mathcal{N}$ with $k_{n} \neq 0$ (and set $\operatorname{argmax}(\mathbf{k})=1$ for $\mathbf{k}=\mathbf{0}$ ). The first step is to observe that it suffices to consider

$$
\mathbf{k} \cdot \boldsymbol{\Omega}+m
$$

with integers $m$ instead of $\mathbf{k} \cdot \boldsymbol{\Omega}+\mathbf{l} \cdot \boldsymbol{\Omega}$, the reason being that $\mathbf{l} \cdot \boldsymbol{\Omega}= \pm \Omega_{n} \pm \Omega_{n^{\prime}}$ is either close to an integer by the asymptotic behaviour $\Omega_{n} \sim n+\lambda \sigma$ of the frequencies (see Lemma 19 below) or may be absorbed into $\mathbf{k} \cdot \boldsymbol{\Omega}$. This is done in Proposition 22 below. Furthermore, if one excludes some values of $\sigma$, a bound of $\mathbf{k} \cdot \boldsymbol{\Omega}+m$ can be obtained from a bound of some derivative

$$
\frac{d^{k}(\mathbf{k} \cdot \boldsymbol{\Omega}+m)}{d \sigma^{k}}
$$

of $\mathbf{k} \cdot \boldsymbol{\Omega}+m$ (Lemma 21). Therefore we study in Lemma 20 a matrix made up of derivatives of the frequencies $\Omega_{n}$. This matrix is such that its inverse multiplied with the vector containing the first derivatives of $\mathbf{k} \cdot \boldsymbol{\Omega}$ is just the vector containing the nonzero entries of $\mathbf{k}$. Bounding its inverse (see Lemma 20) thus helps to study the derivatives of $\mathbf{k} \cdot \boldsymbol{\Omega}+m$.

As in the previous subsection, we fix $\rho_{0}>0$ with (11) and $h$ and $K$ with (12) for some $N \geqslant 2$. Let us emphasize, however, that again all constants will be independent of the discretization parameters $h$ and $K$. We will make extensive use of the asymptotic behaviour of $\mu_{n}$ from (54) and of the modified frequencies described in the following lemma. 
LEMMA 19. We have, for $0 \leqslant \rho \leqslant \rho_{0}$,

$$
n \leqslant \mu_{n} \leqslant C n \quad \text { and } \quad-\frac{C}{n} \leqslant \Omega_{n}-n-\lambda \sigma \leqslant 0 \quad \text { for } n \in \mathcal{N}
$$

with a constant $C=C\left(\sigma_{0}\right)$.

Proof. The estimates of $\mu_{n}$ follow from $n h \leqslant \tan (n h) \leqslant C n h$, by (12). For the estimates of $\Omega_{n}$, we note that

$$
\Omega_{n}-n-\lambda \sigma=\sqrt{\left(\mu_{n}+\lambda \sigma\right)^{2}-\sigma^{2}}-\left(\mu_{n}+\lambda \sigma\right) .
$$

This shows that

$$
0 \geqslant \Omega_{n}-n-\lambda \sigma \geqslant \frac{-\sigma_{0}^{2}}{2 \mu_{n} \sqrt{1+\frac{2 \lambda \sigma}{\mu_{n}}}} .
$$

Estimate (53) of Lemma 18 and $\mu_{n} \geqslant n$ thus lead to the claimed lower bound of $\Omega_{n}-n-\lambda \sigma$.

Now, we begin with the investigation of (integer) linear combinations $\mathbf{k} \cdot \boldsymbol{\Omega}=$ $\sum_{n \in \mathcal{N}} k_{n} \Omega_{n}$ of modified frequencies (58). The following lemma will help us to control derivatives of these linear combinations with respect to $\sigma$, which in turn will allow us to control the linear combinations themselves.

LEMmA 20. Let $1 \leqslant n_{1}<n_{2}<\cdots<n_{M}$, and let $A=\left(a_{k l}\right)_{k, l=1}^{M}$ be the matrix with entries

$$
a_{k l}=\frac{d^{k} \Omega_{n_{l}}(\sigma)}{d \sigma^{k}} .
$$

Then, for all $k, l=1, \ldots, M$ and all $0 \leqslant \sigma \leqslant \sigma_{0}$,

$$
\left|a_{k l}\right| \leqslant C n_{l}^{-k+1} \text { and }\left\|A^{-1}\right\|_{\infty} \leqslant C n_{M}^{2 M}
$$

with a constant $C=C\left(M, \sigma_{0}\right)$.

Proof. We have

$$
a_{k l}=\frac{d^{k} \Omega_{n_{l}}(\sigma)}{d \sigma^{k}}=d_{k} e_{l} x_{l}^{k-1}
$$

with $d_{1}=\lambda$ and $d_{k+1}=-\lambda(2 k-1) d_{k}$ for $k \geqslant 1, e_{l}=1 / \sqrt{1+2 \lambda \sigma / \mu_{n_{l}}}$, and $x_{l}=e_{l}^{2} / \mu_{n_{l}}$. Note that, for all $k, l=1, \ldots, M$,

$$
c^{\prime} \leqslant\left|d_{k}\right| \leqslant C^{\prime}, \quad c^{\prime} \leqslant e_{l} \leqslant C^{\prime} \quad \text { and } \quad \frac{c^{\prime}}{n_{l}} \leqslant x_{l} \leqslant \frac{C^{\prime}}{n_{l}}
$$


with positive constants $c^{\prime}=c^{\prime}\left(\sigma_{0}\right)$ and $C^{\prime}=C^{\prime}\left(M, \sigma_{0}\right)$, by Lemmas 18 and 19. Hence, the bound on the entry $a_{k l}$ as stated in the lemma follows from representation (59).

Moreover, this representation shows that

$$
A=D V E
$$

with diagonal matrices $D=\operatorname{diag}\left(d_{k}\right)_{k=1}^{M}$ and $E=\operatorname{diag}\left(e_{l}\right)_{l=1}^{M}$ and Vandermonde matrix $V=\left(x_{l}^{k-1}\right)_{k, l=1}^{M}$. In order to examine the inverse of $A$, we first invert $V$. Its inverse is given by

$$
V^{-1}=\left(\frac{v_{i j}}{w_{i}}\right)_{i, j=1}^{M}
$$

with

$$
v_{i j}=\sum_{\substack{1 \leqslant l_{1}<\cdots<l_{M-j} \leqslant M \\ l_{1}, \ldots, l_{M-j} \neq i}}(-1)^{j} x_{l_{1}} \cdots x_{l_{M-j}} \text { and } \quad w_{i}=\prod_{\substack{1 \leqslant l \leqslant M \\ l \neq i}}\left(x_{i}-x_{l}\right) ;
$$

see, for example, [23, Section 2.8.1]. Since

$$
\left|x_{i}-x_{l}\right|=x_{i} x_{l}\left|\mu_{n_{l}}-\mu_{n_{i}}\right|=x_{i} x_{l} \frac{\left|n_{l}-n_{i}\right|}{\cos ^{2}(\xi h)}
$$

with $\min \left(n_{i}, n_{l}\right) \leqslant \xi \leqslant \max \left(n_{i}, n_{l}\right)$, the bounds (60) and the step-size restriction (12) imply that

$$
\left\|V^{-1}\right\|_{\infty} \leqslant C n_{M}^{2 M}, \quad\left\|D^{-1}\right\|_{\infty} \leqslant C \quad \text { and } \quad\left\|E^{-1}\right\|_{\infty} \leqslant C
$$

with $C=C\left(M, \sigma_{0}\right)$. The estimate of $\left\|A^{-1}\right\|_{\infty}$ stated in the lemma follows.

Now, we consider sets of values of $\sigma$ for which linear combinations of modified frequencies are small. For vectors $\mathbf{k} \in \mathbb{Z}^{\mathcal{N}}$ and $\mathbf{l} \in \mathbb{Z}^{\mathcal{N}}$, and integers $m$, we define the sets

$$
\mathcal{Q}_{\mathbf{k}, \mathbf{l}, m}(\gamma, \alpha)=\left\{\sigma \in\left[0, \sigma_{0}\right]:|(\mathbf{k}+\mathbf{l}) \cdot \boldsymbol{\Omega}(\sigma)+m|<\frac{\gamma}{\operatorname{argmax}(\mathbf{k})^{\alpha}}\right\} .
$$

We first estimate the Lebesgue measure $|\cdot|$ of these sets in the case when $\mathbf{I}=\mathbf{0}$.

LEMMA 21. There exists a constant $C=C\left(N, \alpha, \sigma_{0}\right)$ such that, for all $0<\gamma \leqslant$ 1 , all $\|\mathbf{k}\| \leqslant N$, and all $m \in \mathbb{Z}$ with $\|\mathbf{k}\|+|m| \neq 0$,

$$
\left|\mathcal{Q}_{\mathbf{k}, \mathbf{0}, m}(\gamma, \alpha)\right| \leqslant \frac{C \gamma^{1 / M}}{\operatorname{argmax}(\mathbf{k})^{\alpha / M-4 M}},
$$

where $M$ denotes the number of nonzero entries of $\mathbf{k}$. 
Proof. We fix a vector $\mathbf{k}$ with $\|\mathbf{k}\| \leqslant N$. We may assume that $\mathbf{k} \neq \mathbf{0}$, because the statement is trivial for $\mathbf{k}=\mathbf{0}$, since $\gamma \leqslant 1$.

(a) Lemma 20 shows that there exists a constant $C=C\left(N, \sigma_{0}\right)$ such that for any $0 \leqslant \sigma \leqslant \sigma_{0}$ there exists $1 \leqslant k \leqslant M$ with

$$
\left|\frac{d^{k}(\mathbf{k} \cdot \boldsymbol{\Omega})(\sigma)}{d \sigma^{k}}\right| \geqslant C \operatorname{argmax}(\mathbf{k})^{-2 M} .
$$

(b) The function $g:\left[0, \sigma_{0}\right] \rightarrow \mathbb{R}, \sigma \mapsto \mathbf{k} \cdot \Omega(\sigma)+m$ is infinitely differentiable, and its first $M+1$ derivatives are uniformly bounded on $\left[0, \sigma_{0}\right]$ by a constant depending only on $\sigma_{0}$ and $N$ (Lemma 20). Property (62) then enables us to apply [2, Lemma 8.4], which yields the statement of the lemma.

Setting

$$
\mathcal{Q}(\gamma, \alpha)=\bigcup_{\substack{\mathbf{k}:\|\mathbf{k}\| \leq N \\ 1:\|1\| \leq 2 \\ \mathbf{k}+\mathbf{1} \neq 0}} \mathcal{Q}_{\mathbf{k}, 1,0}(\gamma, \alpha)
$$

with $\mathcal{Q}_{\mathbf{k}, 1,0}(\gamma, \alpha)$ from (61), we can now prove the following nonresonance result in the spirit of Bambusi's nonresonance condition; see [3, Lemma 5.7].

Proposition 22. Let $\alpha \geqslant(5 N)^{4}$. Then there exists a constant $C=C\left(N, \alpha, \sigma_{0}\right)$ such that, for all $0<\gamma \leqslant 1$,

$$
|\mathcal{Q}(\gamma, \alpha)| \leqslant C \gamma^{1 /(2 \sqrt{\alpha}(N+2))} .
$$

Proof. We consider the sets $\mathcal{Q}_{\mathbf{k}, \mathbf{l}, 0}(\gamma, \alpha)$ for vectors $\mathbf{I}$ with $\|\mathbf{I}\| \leqslant 2$. Throughout this discussion, we fix $0<\gamma \leqslant 1$ and $\mathbf{k}$ with $\|\mathbf{k}\| \leqslant N$.

(a) For the vector $\mathbf{l}=\mathbf{0}$, the measure of the set $\mathcal{Q}_{\mathbf{k}, \mathbf{l}, 0}(\gamma, \alpha)$ can be estimated with Lemma 21.

(b) For $\mathbf{l}= \pm\langle n\rangle$, let $c^{\prime} \geqslant 1$ be a constant such that $\left| \pm \Omega_{n}+\mathbf{k} \cdot \boldsymbol{\Omega}\right| \geqslant 1$ if $n>c^{\prime} \operatorname{argmax}(\mathbf{k})$. This constant exists, by Lemma 19, and it depends on $\sigma_{0}$ and $N$. Then, for $n \leqslant c^{\prime} \operatorname{argmax}(\mathbf{k})$,

$$
\mathcal{Q}_{\mathbf{k}, \mathbf{l}, 0}(\gamma, \alpha) \subseteq \mathcal{Q}_{\mathbf{k}+\mathbf{l}, \mathbf{0}, 0}\left(\left(c^{\prime}\right)^{\alpha} \gamma, \alpha\right)
$$

whereas $\mathcal{Q}_{\mathbf{k}, \mathbf{l}, 0}(\gamma, \alpha)=\emptyset$ for $n>c^{\prime} \operatorname{argmax}(\mathbf{k})$.

(c) For $\mathbf{l}= \pm\left(\langle n\rangle+\left\langle n^{\prime}\right\rangle\right)$, let similarly $c^{\prime \prime}=c^{\prime \prime}\left(N, \sigma_{0}\right) \geqslant 1$ be a constant such that $\left| \pm\left(\Omega_{n}+\Omega_{n^{\prime}}\right)+\mathbf{k} \cdot \boldsymbol{\Omega}\right| \geqslant 1$ if $n+n^{\prime}>c^{\prime \prime} \operatorname{argmax}(\mathbf{k})$. Then, for $n+n^{\prime} \leqslant c^{\prime \prime} \operatorname{argmax}(\mathbf{k})$, 


$$
\mathcal{Q}_{\mathbf{k}, 1,0}(\gamma, \alpha) \subseteq \mathcal{Q}_{\mathbf{k}+\mathbf{l}, \mathbf{0}, 0}\left(\left(c^{\prime \prime}\right)^{\alpha} \gamma, \alpha\right),
$$

whereas $\mathcal{Q}_{\mathbf{k}, 1,0}(\gamma, \alpha)=\emptyset$ for $n+n^{\prime}>c^{\prime \prime} \operatorname{argmax}(\mathbf{k})$.

(d) For $\mathbf{l}= \pm\left(\langle n\rangle-\left\langle n^{\prime}\right\rangle\right)$, where without loss of generality $n<n^{\prime}$, note that, with the constant $C$ of Lemma 19,

$$
|\mathbf{l} \cdot \boldsymbol{\Omega}-m| \leqslant \frac{2 C}{n} \quad \text { for } m= \pm\left(n \pm n^{\prime}\right) .
$$

Then, for $n \geqslant C \operatorname{argmax}(\mathbf{k})^{\sqrt{\alpha}} / \gamma^{1 /(2 \sqrt{\alpha})}$,

$$
\mathcal{Q}_{\mathbf{k}, 1,0}(\gamma, \alpha) \subseteq \mathcal{Q}_{\mathbf{k}, \mathbf{0}, m}\left(3 \gamma^{1 /(2 \sqrt{\alpha})}, \sqrt{\alpha}\right),
$$

and this set is empty for $|m| \geqslant c^{\prime \prime \prime} \operatorname{argmax}(\mathbf{k})$ with a constant $c^{\prime \prime \prime}=c^{\prime \prime \prime}\left(N, \sigma_{0}\right)$, by Lemma 19. On the other hand, we have, for $n<C \operatorname{argmax}(\mathbf{k})^{\sqrt{\alpha}} / \gamma^{1 /(2 \sqrt{\alpha})}$,

$$
\mathcal{Q}_{\mathbf{k}, 1,0}(\gamma, \alpha) \subseteq \mathcal{Q}_{\mathbf{k} \pm\langle n\rangle, \mp\left\langle n^{\prime}\right\rangle, 0}\left(C^{\sqrt{\alpha}} \sqrt{\gamma}, \sqrt{\alpha}\right),
$$

a situation that is covered by (b).

Results (a)-(d) show that there exists a constant $c=c\left(N, \alpha, \sigma_{0}\right)$ such that

$$
\mathcal{Q}(\gamma, \alpha) \subseteq \bigcup_{\substack{\mathbf{k}:\left\|\mathbf{k}|\leqslant N+2 \\ m \in \mathbb{Z}:|||<\left|<c^{\prime \prime \prime r g m a x} \\\|\mathbf{k}|\||+\mid \mathbf{k})\right.\right.}} \mathcal{Q}_{\mathbf{k}, \mathbf{0}, m}\left(c \gamma^{1 /(2 \sqrt{\alpha})}, \sqrt{\alpha}\right) .
$$

Since the number of vectors $\mathbf{k}$ with $\|\mathbf{k}\| \leqslant N+2$ and $\operatorname{argmax}(\mathbf{k})=L$ is at most $(N+3) L^{N+2}$, we have, by Lemma 21 ,

$$
|\mathcal{Q}(\gamma, \alpha)| \leqslant C \gamma^{1 /(2 \sqrt{\alpha}(N+2))} \sum_{L=1}^{\infty} L^{5 N+11-\sqrt{\alpha} /(N+2)}
$$

with a constant $C=C\left(N, \alpha, \sigma_{0}\right)$. The choice of $\alpha$ ensures that $\sqrt{\alpha} \geqslant(N+2)$ $(5 N+13)$, and hence the latter sum converges, and the proposition is proven.

REMARK 23 (Case $\ell \neq 0$ ). For $\ell \neq 0$ but small, the frequencies $\omega_{j}$ from (10) are different from those for $\ell=0$ only for large $j$. For these large $j$, we have to deal with two differences.

First, the frequencies of (10) (and also the modified frequencies) contain an additional summand $\frac{1}{2}|\ell+j \bmod 2 K|^{2}-\frac{1}{2}|\ell-j \bmod 2 K|^{2}$. This is an integer, and it does not affect the proof of Lemmas 20 and 21, where also the integer summand $|j|^{2}-\mu_{|j|^{2}}$ in the modified frequencies (56) does not pose a problem. Nor does it pose a problem in the proof of Proposition 22, since it is of order one for small $\ell$ (for part (b) and (c) of the proof) and is an integer (for part (d) of the proof). 
Second, the quantity $n(j)$ appearing in the frequencies of (10) can be different from $|j|^{2}$. But, for small $\ell$, these two quantities are of the same order; see Lemma 13. We therefore expect the statements of Lemmas 20 and 21 and of Proposition 22 to transfer to this situation with constants depending on $\ell$.

5.4. Proof of Theorem 3. We have already verified in Lemma 18 that Assumption 1 is satisfied under conditions (11) and (12) of Theorem 3. We have also verified in (57) that the modified frequencies (56) are close to the original frequencies, as required in part (i) of Assumption 2. We will now prove that they satisfy the nonresonance condition in part (ii) of Assumption 2 for many values of $h$ and $\rho$. Note that, in the considered case $\ell=0$, part (iii) of Assumption 2 follows from part (ii), since $\varpi_{j}=\varpi_{l}$ for all $j, l \in \mathcal{Z}$ with $n(j)=|j|^{2}=|l|^{2}=n(l)$.

Fix $\rho_{0}>0$ with (11), $h_{0}>0$, and $N$. In contrast to the previous subsection, we do not fix the time step-size $h$ anymore. We consider, for all $0<h \leqslant h_{0}$, the corresponding sets (63) for $\sigma_{0}=\rho_{0}^{2}$, which we denote now by $\mathcal{Q}_{h}(\gamma, \alpha)$ to emphasize the dependence (of the modified frequencies, and hence the sets) on $h$. We set, for $0<\gamma \leqslant 1$,

$$
\mathcal{P}(\gamma)=\left\{(h, \rho) \in\left[0, h_{0}\right] \times\left[0, \rho_{0}\right]: \rho^{2} \notin \mathcal{Q}_{h}(\gamma, \alpha)\right\}
$$

with $\alpha=(5 N)^{4}$. As mentioned above, all $(h, \rho) \in \mathcal{P}(\gamma)$ satisfy Assumption 1 with constant $c_{1}=c_{1}\left(\rho_{0}\right)$ and part (i) of Assumption 2 with $\widehat{\varepsilon}=C_{2} h^{2}$ and constant $C_{2}=C_{2}\left(\rho_{0}\right)$, provided that $K$ satisfies (12).

We still have to show that, for all $(h, \rho) \in \mathcal{P}(\gamma)$, the modified frequencies $\Omega_{n}=\Omega_{n}\left(\rho^{2}\right)$ satisfy the nonresonance condition in part (ii) of Assumption 2 provided that (12) holds. For this purpose, let $\mathbf{k} \in \mathbb{Z}^{\mathcal{N}}$ with $\|\mathbf{k}\| \leqslant N+1$. Then, we have

$$
\frac{2}{\pi}|\mathbf{k} \cdot \boldsymbol{\Omega}| \leqslant\left|\frac{e^{i(\mathbf{k} \cdot \boldsymbol{\Omega}) h}-1}{h}\right|=: \delta,
$$

since the (strong) step-size restriction (12) ensures, together with Lemma 19, that $|\mathbf{k} \cdot \boldsymbol{\Omega}| h \leqslant \pi$. We remark that this is the first and only place where we need that the right-hand side of (12) is $\pi /(N+1)$ and not only $\pi / 3$, say. Now, we write

$$
\mathbf{k} \cdot \boldsymbol{\Omega}= \pm \Omega_{n_{M}} \pm \Omega_{n_{M-1}} \pm \cdots \pm \Omega_{n_{1}}
$$

with $n_{M} \geqslant n_{M-1} \geqslant \cdots \geqslant n_{1}$ in such a way that there is no pairwise cancellation $(M=\|\mathbf{k}\|)$. For $\delta \leqslant 1$, we have, by (64) and by Lemma 19 , that $n_{M} \leqslant c n_{M-1}$ with $c=c\left(N \rho_{0}\right)$. Moreover, the choice of the set $\mathcal{Q}_{h}(\gamma, \alpha)$ yields

$$
|\mathbf{k} \cdot \boldsymbol{\Omega}| \geqslant \gamma\left(\frac{n_{M}^{2}}{c n_{M} n_{M-1} \cdots n_{1}}\right)^{\alpha} .
$$


Combining this with (64), we get

$$
\left(\frac{n_{M}^{2}}{\prod_{n \in \mathcal{N}} n^{\left|k_{n}\right|}}\right)^{\alpha} \leqslant \frac{c^{\alpha} \pi}{2 \gamma} \delta
$$

The nonresonance condition of Assumption 2 thus holds for $c_{2}=c_{2}\left(N, \gamma, \rho_{0}\right)$, $\delta_{2}=1$, and $s_{2}=\alpha N$.

We finally have to estimate the Lebesgue measure of $\mathcal{P}(\gamma)$. By Fubini's theorem and Proposition 22, we have

$$
|\mathcal{P}(\gamma)|=\rho_{0} h_{0}-\int_{0}^{h_{0}} \mathcal{Q}_{h}(\gamma, \alpha) d h \geqslant \rho_{0} h_{0}-C h_{0} \gamma^{1 /(2 \sqrt{\alpha}(N+2))},
$$

because the constant in this proposition is independent of $h$. The proof of Theorem 3 is thus complete, if we redefine $\gamma$.

\section{References}

[1] G. P. Agrawal, Nonlinear Fiber Optics, 5th ed. (Academic Press, 2013).

[2] D. Bambusi, 'On long time stability in Hamiltonian perturbations of nonresonant linear PDEs', Nonlinearity 12 (1999), 823-850.

[3] D. Bambusi and B. Grébert, 'Birkhoff normal form for partial differential equations with tame modulus', Duke Math. J. 135 (2006), 507-567.

[4] B. Cano and A. González-Pachón, Plane waves numerical stability of some explicit exponential methods for cubic Schrödinger equation, Preprint, 2013. http://hermite.mac.cie .uva.es/bego/cgp3.pdf.

[5] D. Cohen, E. Hairer and Ch. Lubich, 'Long-time analysis of nonlinearly perturbed wave equations via modulated Fourier expansions', Arch. Ration. Mech. Anal. 187 (2008), 341-368.

[6] M. Dahlby and B. Owren, 'Plane wave stability of some conservative schemes for the cubic Schrödinger equation', M2AN Math. Model. Numer. Anal. 43 (2009), 677-687.

[7] E. Faou, Geometric Numerical Integration and Schrödinger Equations, Zurich Lectures in Advanced Mathematics (European Mathematical Society (EMS), Zürich, 2012).

[8] E. Faou, L. Gauckler and Ch. Lubich, 'Sobolev stability of plane wave solutions to the cubic nonlinear Schrödinger equations on a torus', Comm. Partial Differential Equations 38 (2013), 1123-1140.

[9] E. Faou, B. Grébert and E. Paturel, 'Birkhoff normal form for splitting methods applied to semilinear Hamiltonian PDEs. I. Finite-dimensional discretization', Numer. Math. 114 (2010), 429-458.

[10] E. Faou, B. Grébert and E. Paturel, 'Birkhoff normal form for splitting methods applied to semilinear Hamiltonian PDEs. II. Abstract splitting', Numer. Math. 114 (2010), 459-490.

[11] L. Gauckler, Long-time analysis of Hamiltonian partial differential equations and their discretizations, Dissertation (Doctoral Thesis), Universität Tübingen, 2010, http://nbn-resol ving.de/urn:nbn:de:bsz:21-opus-47540. 
[12] L. Gauckler, E. Hairer and Ch. Lubich, 'Energy separation in oscillatory Hamiltonian systems without any non-resonance condition', Comm. Math. Phys. 321 (2013), 803-815.

[13] L. Gauckler and Ch. Lubich, 'Splitting integrators for nonlinear Schrödinger equations over long times', Found. Comput. Math. 10 (2010), 275-302.

[14] L. Gauckler, E. Hairer, Ch. Lubich and D. Weiss, 'Metastable energy strata in weakly nonlinear wave equations', Comm. Partial Differential Equations 37 (2012), 1391-1413.

[15] E. Hairer and Ch. Lubich, 'Long-time energy conservation of numerical methods for oscillatory differential equations', SIAM J. Numer. Anal. 38 (2000), 414-441. (electronic).

[16] E. Hairer and Ch. Lubich, 'On the energy distribution in Fermi-Pasta-Ulam lattices', Arch. Ration. Mech. Anal. 205 (2012), 993-1029.

[17] E. Hairer, Ch. Lubich and G. Wanner, Geometric Numerical Integration. Structure-Preserving Algorithms for Ordinary Differential Equations, 2nd ed, Springer Series in Computational Mathematics vol. 31 (Springer-Verlag, Berlin, 2006).

[18] Z. Hani, 'Long-time instability and unbounded Sobolev orbits for some periodic nonlinear Schrödinger equations', Arch. Ration. Mech. Anal. 211 (2014), 929-964.

[19] R. H. Hardin and F. D. Tappert, 'Applications of the split-step Fourier method to the numerical solution of nonlinear and variable coefficient wave equations', SIAM Rev. 15 (1973), 423.

[20] S. Jin, P. Markowich and Ch. Sparber, 'Mathematical and computational methods for semiclassical Schrödinger equations', Acta Numer. 20 (2011), 121-209.

[21] M. Khanamiryan, O. Nevanlinna and T. Vesanen, Long-term behavior of the numerical solution of the cubic nonlinear Schrödinger equation using Strang splitting method, Preprint, 2012. http://www.damtp.cam.ac.uk/user/na/people/Marianna/papers/NLS.pdf.

[22] T. I. Lakoba, 'Instability of the split-step method for a signal with nonzero central frequency', J. Opt. Soc. Am. B 30 (2013), 3260-3271.

[23] W. H. Press, S. A. Teukolsky, W. T. Vetterling and B. P. Flannery, Numerical Recipes. The Art of Scientific Computing, 3rd ed. (Cambridge University Press, Cambridge, 2007).

[24] T. R. Taha and M. J. Ablowitz, 'Analytical and numerical aspects of certain nonlinear evolution equations. II. Numerical, nonlinear Schrödinger equation', J. Comput. Phys. 55 (1984), 203-230.

[25] J. A. C. Weideman and B. M. Herbst, 'Split-step methods for the solution of the nonlinear Schrödinger equation', SIAM J. Numer. Anal. 23 (1986), 485-507. 